\title{
Near infrared flares of Sagittarius $A^{*}$ Importance of near infrared polarimetry
}

\author{
M. Zamaninasab ${ }^{1,2}$, A. Eckart ${ }^{1,2}$, G. Witzel ${ }^{1}$, M. Dovciak ${ }^{3}$, V. Karas ${ }^{3}$, R. Schödel ${ }^{4}$, R. Gießübel ${ }^{1,2}$, M. Bremer ${ }^{1}$, \\ M. García-Marín ${ }^{1}$, D. Kunneriath ${ }^{1,2}$, K. Mužić ${ }^{1}$, S. Nishiyama ${ }^{5}$, N. Sabha ${ }^{1}$, C. Straubmeier ${ }^{1}$, and A. Zensus ${ }^{2,1}$ \\ 1 I.Physikalisches Institut, Universität zu Köln, Zülpicher Str.77, 50937 Köln, Germany \\ e-mail: zamani@ph1.uni-koeln.de \\ 2 Max-Planck-Institut für Radioastronomie, Auf dem Hügel 69, 53121 Bonn, Germany \\ 3 Astronomical Institute, Academy of Sciences, Boční II, 14131 Prague, Czech Republic \\ ${ }^{4}$ Instituto de Astrofísica de Andalucía -CSIC, Glorieta de la Astronomía S/N, 18008 Granada, Spain \\ 5 Department of Astronomy, Kyoto University, Kyoto 606-8502, Japan
}

Received 12 May 2009 / Accepted 8 November 2009

\section{ABSTRACT}

\begin{abstract}
Context. We report on the results of new simulations of near-infrared (NIR) observations of the Sagittarius A* (Sgr A*) counterpart associated with the super-massive black hole at the Galactic Center.

Aims. Our goal is to investigate and understand the physical processes behind the variability associated with the NIR flaring emission from Sgr A*.

Methods. The observations have been carried out using the NACO adaptive optics (AO) instrument at the European Southern Observatory's Very Large Telescope and CIAO NIR camera on the Subaru telescope (13 june 2004; 30 july 2005; 1 june 2006; 15 may 2007; 17 may 2007 and 28 may 2008). We used a model of synchrotron emission from relativistic electrons in the inner parts of an accretion disk. The relativistic simulations have been carried out using the Karas-Yaqoob (KY) ray-tracing code.

Results. We probe the existence of a correlation between the modulations of the observed flux density light curves and changes in polarimetric data. Furthermore, we confirm that the same correlation is also predicted by the hot spot model. Correlations between intensity and polarimetric parameters of the observed light curves as well as a comparison of predicted and observed light curve features through a pattern recognition algorithm result in the detection of a signature of orbiting matter under the influence of strong gravity. This pattern is detected statistically significant against randomly polarized red noise. Expected results from future observations of VLT interferometry like GRAVITY experiment are also discussed.

Conclusions. The observed correlations between flux modulations and changes in linear polarization degree and angle can be a sign that the NIR flares have properties that are not expected from purely random red-noise. We find that the geometric shape of the emission region plays a major role in the predictions of the model. From fully relativistic simulations of a spiral shape emitting region, we conclude that the observed swings of the polarization angle during NIR flares support the idea of compact orbiting spots instead of extended patterns. The effects of gravitational shearing, fast synchrotron cooling of the components and confusion from a variable accretion disk have been taken into account. Simulated centroids of NIR images lead us to the conclusion that a clear observation of the position wander of the center of NIR images with future infrared interferometers will prove the existence of orbiting hot spots in the vicinity of our Galactic super-massive black hole.
\end{abstract}

Key words. black hole physics - infrared: general - accretion, accretion disks - Galaxy: center - Galaxy: nucleus

\section{Introduction}

The nearest super-massive black hole candidate $\left(\sim 4 \times 10^{6} M_{\odot}\right)$ lies at the center of our galaxy, as inferred from motions of stars near the Galactic Center (Eckart \& Genzel 1996, 1997; Eckart et al. 2002; Schödel et al. 2002; Eisenhauer et al. 2003; Ghez et al. 2000, 2005, 2008; Gillessen et al. 2009). With a luminosity of $10^{-9}-10^{-10} L_{\text {Edd }}$, where $L_{\text {Edd }}$ is its limiting Eddington luminosity, Sagittarius $A^{*}$, the radio source associated with this $\mathrm{SMBH}$, is one of the most extreme sub-Eddington sources accessible to observations. However, X-ray and near-infrared (NIR) flares are routinely detected with high spatial and spectral resolution observations (Baganoff et al. 2001; Porquet et al. 2003, 2008; Genzel et al. 2003; Eckart et al. 2004, 2006a-c, 2008a-c; Meyer et al. 2006a,b, 2007; Yusef-Zadeh et al. 2006a,b, 2007, 2008). These short bursts of increased radiation last normally for about $100 \mathrm{~min}$ and occur four to five times a day (see Fig. 1 for a typical behavior of Sgr A* during a flaring phase in NIR bandwidth).

Recent NIR and X-ray observations have revealed the nonthermal nature of high frequency radiation from Sgr A* (Eckart et al. 2006a-c, 2008a-c; Gillessen et al. 2006; Hornstein et al. 2007). Sgr A* is probably visible in the NIR regime only during its flaring state. The short time scale variabilities seen during several observed NIR and X-ray flares argue for an emitting region not bigger than about ten Schwarzschild radii $\left(r_{\mathrm{s}}=\frac{2 G M}{c^{2}}=\right.$ $\left.2 r_{\mathrm{g}}=1.2 \times 10^{12}\left(\frac{M}{4 \times 10^{6} M_{\odot}}\right) \mathrm{cm}\right)$ of the associated super-massive black hole (Baganoff et al. 2001; Genzel et al. 2003). We have scaled the relevant physical distances according to the gravitational radius $\left(r_{\mathrm{g}}\right)$ throughout this paper. The NIR flares are highly polarized and normally have X-ray counterparts, which strongly suggests a synchrotron-self-Compton (SSC) or inverse Compton emission as the responsible radiation mechanism 


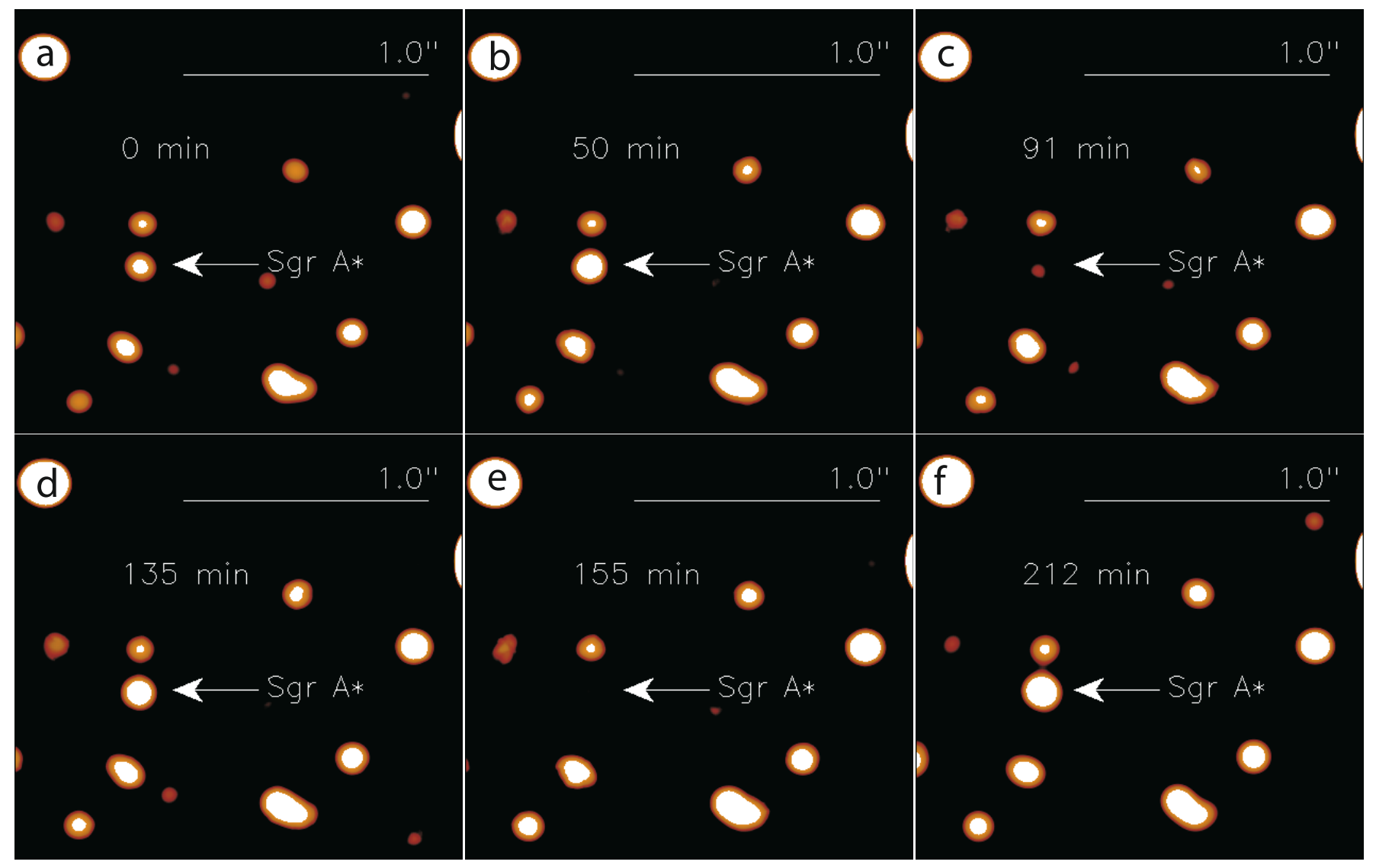

Fig. 1. Sgr A* as it was observed in NIR $L^{\prime}$-band $(3.8 \mu \mathrm{m})$ on 3 june 2008 between 05:29:00 - 09:42:00 (UT time). (a)-f)) show the observed images of Sgr A* after 0, 50, 91, 135, 155 and 212 min off the start of observation. The images show that when Sgr A* is in its flaring state the flux changes up to $100 \%$ in time intervals of the order of only tens of minutes.

(Eckart et al. 2004, 2006a,b; Yuan et al. 2004; Liu et al. 2006). Several observations have already confirmed the existence of a time lag between the simultaneous NIR/X-ray flares and the flares in the lower frequencies. This is interpreted as a sign for cooling down via adiabatic expansion (Eckart et al. 2006a, 2008b,c; Yusef-Zadeh et al. 2006a,b, 2007, 2008; Marrone et al. 2008, Zamaninasab et al. 2008a).

The other feature related to these NIR/X-ray flares are the claimed quasi-periodic oscillations (QPOs) with a period of $20 \pm 5 \mathrm{~min}$, which have been reported in several of these events (Genzel et al. 2003; Belanger et al. 2006; Eckart et al. 2006b,c; 2008a; Meyer et al. 2006a,b, Hamaus et al. (2009)). Short periods of increased radiation (the so called "NIR flares", normally around $100 \mathrm{~min}$ ) seem to be accompanied by QPOs. All the studies mentioned above probed this $20 \pm 5$ min quasi-periodicity, by performing a sliding window analysis with window lengths of the order of the flaring time. Recently, Do et al. (2009) argued that they did not find any significant periodicity at any time scale while probing their sample of observations for a periodic signal. Their method is based on the Lomb-Scargle periodogram analysis of a sample of six light curves and comparing them with several thousands of artificial light curves with the same underlying red-noise. One must note that the suggested QPOs are transient phenomena, lasting for only very few cycles (50$100 \mathrm{~min}$ ). This kind of behavior, along with the inevitable uncertainty in the red noise power law index determination, makes a clear and unambiguous detection $(>5 \sigma)$ of a periodic signal very difficult. Whenever a flare of Sgr A* was observed with polarimetry, it was found that it is accompanied by significant polarization which varies on similarly short timescales as the light curve itself. By carrying out an analysis only on the total flux, some pieces of the observed information are ignored. It is already known that polarimetric data have been shown to be able to reveal substructure in flares, even when the light curve appears largely featureless (e.g. see Fig. 4 in Eckart et al. 2006b). The other main advantage of polarimetric observations is that, in addition to the flux density light curve, one can analyze the changes in the observed degree of polarization and the changes in polarization angle during flaring time as well.

The claimed quasi-periodicity has been interpreted as being related to the orbital time scale of the matter in the inner parts of the accretion disk. According to well-known observed high frequency quasi-periodic oscillations (HFQPOs) in X-ray light curves of stellar mass black holes and binaries (Nowak \& Lehr 1998), this interpretation is of special interest since it shows a way to better understand the behavior of accretion disks for a wide range of black hole masses. The recent unambiguous discovery of a one hour (quasi-)periodicity in the X-ray emission light curve of the active galaxy RE J1034+396 provides further support to this idea and extends the similarity between stellarmass and super-massive black holes to a new territory (Gierliński et al. 2008).

Although the origin of the observed QPOs in the sources associated with black holes is still a matter of debate, several magnetohydrodynamic (MHD) simulations confirmed that it could be related to instabilities in the inner parts of the accretion disks, very close to the marginally stable orbit of the black hole $\left(r_{\mathrm{mso}}\right)$, and also possibly connected with the so-called "stress edge" 
Table 1. Observations log.

\begin{tabular}{lccccccc}
\hline \hline Date (Telescope) & Spectral domain & $\begin{array}{c}\text { UT start } \\
\text { time }\end{array}$ & $\begin{array}{c}\text { UT stop } \\
\text { time }\end{array}$ & Max. flux & Min. flux & $\begin{array}{c}\text { Average flux } \\
\text { sampling rate }\end{array}$ & $\begin{array}{c}\text { Average polarization } \\
\text { sampling rate }\end{array}$ \\
\hline 2004 June 13 (NACO) & $2.2 \mu \mathrm{m}$ & $07: 20: 02$ & $09: 15: 08$ & $5.19 \mathrm{mJy}$ & $2.21 \mathrm{mJy}$ & $1.2 \mathrm{~min}$ & $3 \mathrm{~min}$ \\
2005 July 30 (NACO) & $2.2 \mu \mathrm{m}$ & $02: 07: 36$ & $07: 03: 39$ & $8.19 \mathrm{mJy}$ & $1.23 \mathrm{mJy}$ & $1.2 \mathrm{~min}$ & $3 \mathrm{~min}$ \\
2006 June 1 (NACO) & $2.2 \mu \mathrm{m}$ & $04: 26: 03$ & $10: 44: 27$ & $19.33 \mathrm{mJy}$ & $0.72 \mathrm{mJy}$ & $1.5 \mathrm{~min}$ & $2 \mathrm{~min}$ \\
2007 May 15 (NACO) & $2.2 \mu \mathrm{m}$ & $09: 08: 14$ & $09: 42: 12$ & $22.27 \mathrm{mJy}$ & $1.70 \mathrm{mJy}$ & $1.5 \mathrm{~min}$ & $2 \mathrm{~min}$ \\
2007 May 17 (NACO) & $2.2 \mu \mathrm{m}$ & $04: 42: 14$ & $09: 34: 40$ & $11.87 \mathrm{mJy}$ & $1.86 \mathrm{mJy}$ & $1.5 \mathrm{~min}$ & $2 \mathrm{~min}$ \\
2008 May 28 (CIAO) & $2.15 \mu \mathrm{m}$ & $09: 22: 51$ & $13: 00: 37$ & $7.70 \mathrm{mJy}$ & $0.97 \mathrm{mJy}$ & $3.3 \mathrm{~min}$ & $10 \mathrm{~min}$ \\
\hline
\end{tabular}

(Hawley 1991; Chan et al. 2009b). If the flux modulations are related to a single azimuthal compact over-dense region (hereafter: "hot spot"), orbiting with the same speed as the underlying accretion disk, one can constrain the spin of the black hole by connecting the observed time scales of QPOs to the orbital time scale of matter around the black hole: $T=2.07\left(r^{\frac{3}{2}}+\right.$ a) $\left(\frac{M}{4 \times 10^{6} M_{\odot}}\right)$ min (Bardeen et al. 1972, where $-1 \leq a \leq 1$ is the black hole dimensionless spin parameter and $r$ is the distance of the spot from the black hole). The characteristic behavior of general relativistic flux modulations produced via the orbiting hot spots have been discussed in several papers (see e.g. Cunningham \& Bardeen 1973; Abramowicz et al. 1991; Karas \& Bao 1992; Hollywood et al. 1995; Dovčiak 2004, 2007). In this paper, we have used a spotted accretion disk scenario to model the observed patterns of our sample of NIR light curves.

Several authors have proposed different models in order to explain the flaring activity of Sgr A*. These models cover a wide range of hypotheses like disk-star interactions (Nayakshin et al. 2004), stochastic acceleration of electrons in the inner region of the disk (Liu et al. 2006), sudden changes in the accretion rate of the black hole (Liu et al. 2002), heating of electrons close to the core of a jet (Markoff et al. 2001; Yuan et al. 2002), trapped oscillatory modes in the inner regions of the accretion disk in the form of spiral patterns or Rossby waves (Tagger \& Melia 2006; Falanga et al. 2007; Karas et al. 2008), non-axisymmetric density perturbations which emerge as the disk evolves in time (Chan et al. 2009b), non-Keplerian orbiting spots falling inward inside the plunging region created via magnetic reconnections (Falanga et al. 2008), and also comet like objects trapped and tidally disrupted by the black hole (Čadež et al. 2006; Kostić et al. 2009).

Observational data render some of these models unlikely. The star-disk interaction model is unable to produce the repeated flux modulations and also the high degree of polarization since it mainly deals with thermal emission. Tidal disruption of cometlike objects are also unable to reproduce the observed rate of flares per day, since the estimated capture rate of such objects for the Sgr A* environment is at least one order of magnitude lower (Čadež et al. 2006; this is a very rough estimate. The actual capture rate of such objects can not be well determined (A. Čadež, private communication)). Nevertheless, several viable models exist and make different predictions that can be distinguished observationally. For example, one important characteristic prediction of hot spot models is about the wobbling of the center of the images (Broderick \& Loeb 2006a,b; Paumard et al. 2006; Zamaninasab et al. 2008b; Hamaus et al. 2009). Significant effort has been already devoted to measure this possible position wander of Sgr A* in the mm, sub-mm and NIR regimes (Eisenhauer 2005b, 2008; Gillessen 2006; Reid et al. 2008; Doelleman et al. 2008). In this paper we discuss how NIR polarimetry and the next generations of VLT interferometry (VLTI) and Very Long Baseline Interferometry (VLBI) experiments can provide data to support or reject certain models for the accretion flow/outflow related to Sgr A*. Obtaining accurate data on the accretion flow of Sgr A* can lead us to a better understanding of the physics of strong gravitational regimes, formation of black holes and their possible relation to the galaxy formation process in a cosmological context.

In Sect. 2 we present a complete sample of NIR light curves observed in the polarimetry mode. A brief description about the details of the observation and data reduction methods is provided. We discuss the quasi-periodicity detection methods and present the results of a correlation analysis between the flux and polarimetric parameters. A general description of our model setup and results of simulations are discussed in Sect. 3. We show how NIR polarimetry can be used as a way to constrain physical parameters of the emitting region (like its geometrical shape) in Sect. 4 and Sect. 5. In Sect. 6 we mainly discuss the predictions that the future NIR interferometer (GRAVITY) is expected to reveal and how different assumptions in the model parameters can modify the results. In Sect. 7 we summarize the main results of the paper and draw our conclusions.

\section{NIR polarimetry}

\subsection{Observations and data reduction}

All observations we refer to in this paper have been carried out in the NIR $K_{s}$ band with the NIR camera CONICA and the adaptive optics (AO) module NAOS (NACO) at the ESO VLT unit telescope 4 (YEPUN) on Paranal, Chile ${ }^{1}$ and CIAO NIR camera on the Subaru telescope ${ }^{2}$. These facilities are suited for both time resolved observations of total intensity and polarimetric degree and angle with a sampling of about two to three minutes. The NAOS/CONICA NIR camera installed on UT4, VLT allows for a simultaneous measurement of two orthogonal directions of electric field vector via a Wollaston prism. The combination with a rotary half-wave plate allows the rapid alternation between measurements of different angles of the electric vector. This is crucial for determining the linear polarization characteristics of a time-varying source. The CIAO camera uses a rotating half-wave plane combined with a fixed wire grid polarizer for measuring the linear polarization.

Since the first NIR polarimetric observation of $\mathrm{Sgr} \mathrm{A}^{*}$ in 2004 (Eckart et al. 2006a), several polarized flares have been observed (Meyer et al. 2006a,b, 2007; Eckart et al. 2008a). In

\footnotetext{
1 Based on observations at the Very Large Telescope (VLT) of the European Southern Observatory (ESO) on Paranal in Chile; Programs:075.B-0093 and 271.B-5019(A).

2 Based on data collected at Subaru Telescope, which is operated by the National Astronomical Observatory of Japan.
} 
the VLT observations, the infrared wavefront sensor of NAOS was used to lock the AO loop on the NIR bright ( $K$-band magnitude $~ 6.5$ ) supergiant IRS 7, located about 5.6" north of Sgr A*. Atmospheric conditions (and consequently the AO correction) were stable enough during the observations for doing high angular resolution photometry and polarimetry (with a typical coherence time of 2 milliseconds and larger). The exposures have been dithered. The reductions of the 1 june 2006 and 15 may 2007 data presented here have been repeated for this publication to confirm the significance of the discussed features (see also Eckart et al. 2008a). The 17 may 2007 data have not been published before. All exposures were sky subtracted, flatfielded, and corrected for dead or bad pixels. As the most important improvement of the new reduction the dithered exposures have been aligned with sub-pixel accuracy by a cross-correlation method (Devillard 1999). PSFs were extracted from these images with StarFinder (Diolaiti et al. 2000). The images were deconvolved with the Lucy-Richardson (LR) algorithm. Beam restoration was carried out with a Gaussian beam of $F W H M$ corresponding to the respective wavelength. The final resolution at $2.2 \mu \mathrm{m}$ is about 60 milli-arcseconds (mas). Flux densities of the sources were measured by aperture photometry. Because of the high accuracy of the image alignment it was possible to separate Sgr A* from the nearby stars S17 and S13 by choosing a circular aperture of about 52 mas radius (see Fig. 15 of Eckart et al. 2006a, and discussion therein), resulting in a better correction for the flux contribution of these stars. The data was corrected for extinction using $A_{K}=2.8$ (Eisenhauer et al. 2005a; Schödel et al. 2007). The relative flux density calibration was carried out using known $K$-band flux densities and positions of 14 sources in the IRS16 cluster by R. Schödel (private communications). This results in a $K$-band flux of the high velocity star $\mathrm{S} 2$ of $22 \pm 1 \mathrm{mJy}$, which compares well with the magnitudes and fluxes for S2 quoted by Ghez et al. (2005b) and Genzel et al. (2003). The measurement uncertainties for Sgr A* were obtained from the reference star S2. For more details about CIAO observations see Nishiyama et al. (2009).

\subsection{Data analysis}

\subsubsection{Periodicity}

Figure 2 shows flare events observed in the NIR $K$-band $(2.2 \mu \mathrm{m})$ on 13 june 2004; 30 july 2005; 1 june 2006; 15 may 2007; 17 may 2007 and 28 may 2008. This sample includes all flare events observed in NIR polarimetry during recent years according to the knowledge of the authors. The flux densities rise and come back to their quiescent level in time intervals of roughly $100 \mathrm{~min}$. The measured values of total flux, degree of polarization, and angle of polarization vary significantly on time scales of $\sim 10 \mathrm{~min}$. These abrupt changes can be more clearly detected when the events are in their brightest state. Even if the flares are different in some aspects (e.g. the ratio of the changes of the flux, the maximum brightness achieved, or the degree of linear polarization), there could exist some features that are repeated in our sample. Here we perform a quantitative analysis in order to probe such features. We focus first on detecting periodic signatures in flux densities and then a possible correlation between changes in flux and polarimetric light curves.

The autocorrelation function and Lomb-Scargle periodograms can be used to detect signatures of time periodic structures. For the autocorrelation analysis we used the $\mathrm{z}$ transformed discrete correlation function (ZDCF) algorithm (Alexander 1997), which is particularly useful for analyzing sparse, unevenly sampled light curves. For Lomb-Scargle periodograms we followed Press \& Rybicki (1989).

Figure 3 shows the cross-correlation of the flux density light curves of our sample with themselves by using ZDCF method. The ZDCF of the 30 july 2005; 1 june 2006; 15 and 17 may 2007 flares show peaks around $20 \pm 5$ min time-lag, and specially for 15 and 17 may 2007 the peaks look significant. These peaks can be signs of a possible periodicity.

As one can see, only 17 may 2007's ZDCF shows a peak above the $99.8 \%$ significance threshold. The false alarm values have been derived by repeating the same ZDCF analysis on the $10^{4}$ random red-noise light curves. The criteria for this comparison and the way the light curves are produced are described below. Here we must note that since the ZDCF values are normalized and distributed in a [-1:1] interval, the distribution of the values for each time lag $(\tau)$ is not Gaussian (see Fig. 4). As a result we have used the median and percentile nomenculture instead of the normal standard deviation formalism for deriving the false alarm levels.

We have highlighted the time windows in which ZDCFs show a peak around $20 \pm 5 \mathrm{~min}$, coinciding with the claimed quasi-periodicity. Even though the 1 june 2006 and 15 may 2007's ZDCFs do not show peaks above $99.8 \%$ significance, they however still reach $99 \%$ and $95.4 \%$ levels, respectively. Also, by a rough estimate, the significance of the ZDCF peaks are correlated to the brightness of the flares, and for the faint events, as is observed for example in 2004, there is no detectable peak.

Figure 5 shows the Lomb-Scargle periodogram for each flux light curve. The usual factor of four times over-sampling was applied in order to increase the sensitivity. All periodograms show the power law behavior, $\mathcal{P} \propto f^{\omega}$, showing greater power at lower frequencies. Here $\mathcal{P}$ is power, $f$ is frequency and $\omega$ is power law index. All light curves follow a red noise under-lying power spectral density (PSD) with $-1.19 \leq \omega \leq-1.77$. To test the significance of the peaks in the periodogram we have repeated the Lomb-Scargle analysis for $10^{4}$ simulated red noise light curves. For simulating the red noise we have followed the algorithm by Trimmer and König (1995) (see Figs. 9-11 for examples). The red noise light curves were produced for each event separately following the procedure below: (1) Determining the slope of the observed PSD for each event using a linear fit to the periodogram in log-log space and also by fitting a first-order autoregressive function to the PSD (Schulz \& Mudelsee 2002) and averaging the results of both methods (the resulted $\omega$ is presented in the lower right corner of each plot in Fig. 5). (2) $10^{4}$ red noise light curves produced with the related $\omega$ following the method of Timmer \& König (1995). The light curves have been produced by selecting a middle part of a light curve with a length at least ten times longer than the observed light curve following the argument by Uttley et al. (2002). The selected segment of the light curve is sampled to the same time bin of the corresponding observation. This will correct for any artificial effects caused by time lags which exist on our light curves and also the uneven sampling of our observations. (3) The value of the flux has multiplied by one factor as the mean of the simulated flux has the same value as the observational one for each night.

None of the periodograms of Fig. 5 show a peak that exceeds the $99.8 \%$ threshold except the ones correlated to the lengths of the flare events $(\sim 100 \mathrm{~min})$. We specially highlighted the windows in which corresponding ZDCFs peak. In order to see if there exists a persistent frequency peak in the PSDs, we averaged all observed periodograms (Fig. 6b). The average periodogram doesn't show a significant peak in comparison with the 

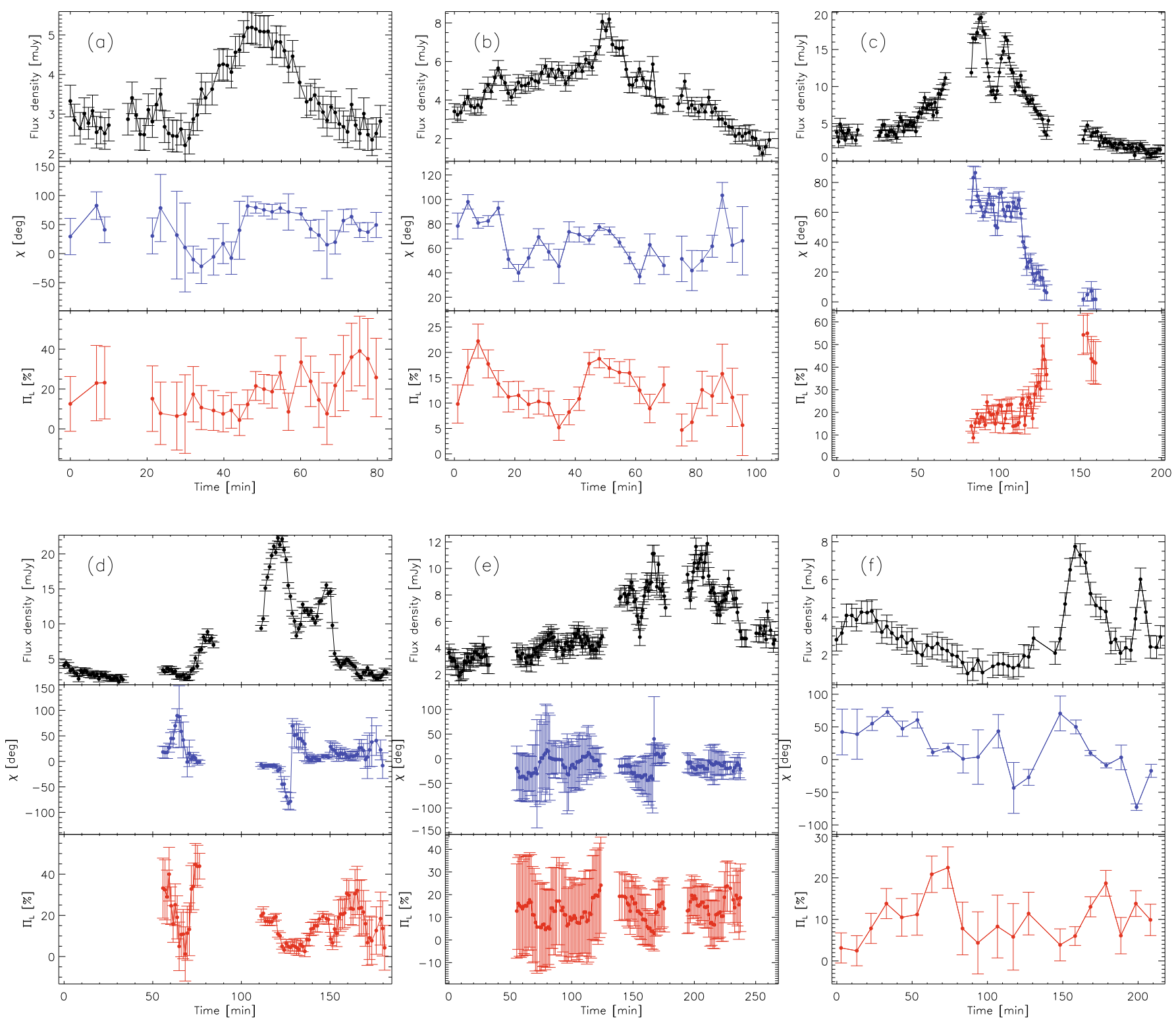

Fig. 2. Our sample of light curves of Sgr A* flares observed in NIR $K_{s}$ band $(2.2 \mu \mathrm{m})$ polarimetry mode. The events were observed on 2004 june 13 a); 2005 july 30 b); 2006 june 1 c); 2007 may 15 d); 2007 may 17 e) and 2008 may 28 f). In each panel, the top shows the de-reddened flux density measured in mJy (black), the middle shows the polarization angle (blue) and the bottom shows the degree of linear polarization (red). The gaps in the light curves are due to the sky background measurements.

false alarm level even if we exclude the 13 june 2004 light curve (Fig. 6c). As we mentioned before the short time scale of the flares and limited number of cycles make a significant detection of any periodicity very difficult. In addition, the possible quasiperiodic structure can vary during the time of the flare if it is connected to the falling clumps of matter into the black hole (Falanga et al. 2008). In this case the Lomb-Scargle algorithm finds different frequencies and allocates them separate values of power. This effect can result in a periodogram in which the values of power are higher in a band of frequencies instead of a specific value. As one can see in Fig. 5, 1 june 2006 and 15 may 2007 can be the candidates for such an effect since the value of the PSD function remains high in the highlighted window. To test for such an effect we averaged the periodograms with a new bin size and repeated the same procedure for the random red noise PSDs (Fig. 7). The resulting averaged periodogram does not show any significant peak again (Fig. 7b), even if we exclude the 13 june 2004 event (Fig. 7c). Since this procedure is very sensitive to the size of the chosen window (which can vary from event to event), a more detailed analysis is needed to study the possible evolution of any periodic signal.

\subsubsection{Importance of polarimetry}

We have also performed cross correlation analysis between variations of flux and degree (angle) of polarization. A search for any short time-lag correlation has been carried out by scanning the light curves using a sliding window method (see Fig. 12a). The size of the scanning window $(\epsilon)$ is fixed on 40 min since we are interested in magnifying any short lag correlation related to the possible $20 \pm 5$ min quasi-periodicity. Figure 8 shows the cross-correlations of the two sets of mentioned light curves for all events in our sample. The time steps of the scans were fixed to be five minutes. The presented results are the average of all scans weighted by the flare signal to noise ratio (i.e. each part of 

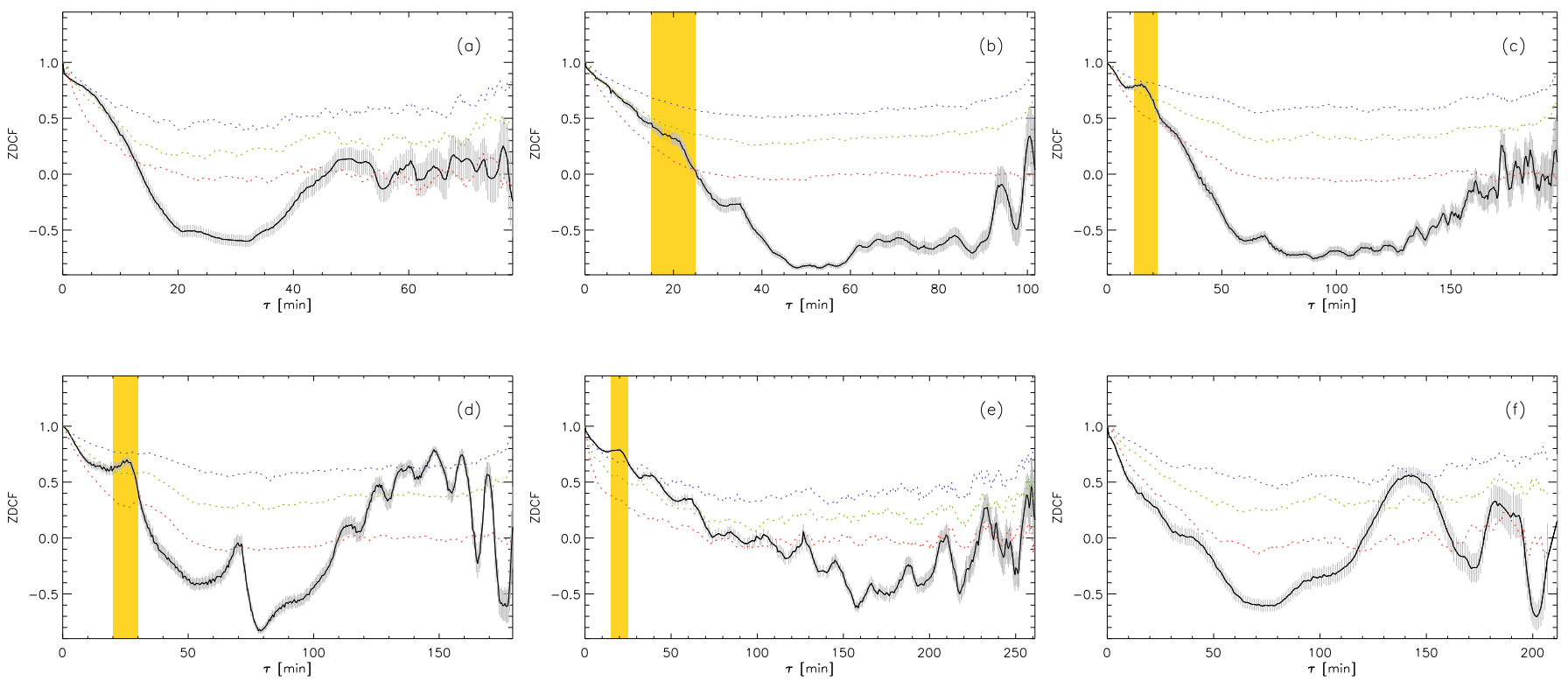

Fig. 3. ZDCF of the flux light curves of 13 june 2004 a); 30 july 2005 b); 1 june 2006 c); 15 may 2007 d); 17 may 2007 e) and 28 may 2008 f). The vertical colored boxes indicate the position of the closest peaks to the zero time lags. Dotted lines show the median (red), $68.3 \%$ (green) and $99.8 \%$ (blue) thresholds derived from $10^{4}$ red noise simulated light curves.
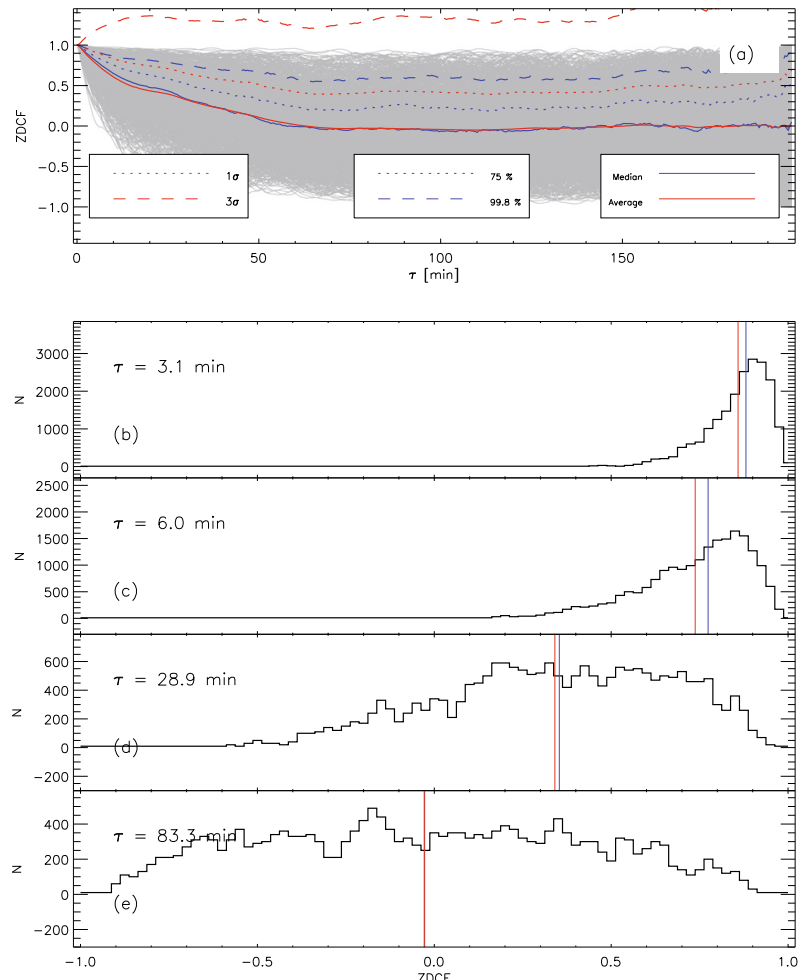

Fig. 4. ZDCF of $10^{4}$ simulated red noise light curves overplotted in one image a). The resulted average and standard deviation as well as the median and percentile values are presented. The distribution of the ZDCF values for different time lags $(\tau)$ are also depicted (b)-e)), which clearly show non-Gaussian distributions, specially for small values of $\tau$.

the flare that is brighter, has more weight). Since the ZDCF algorithm needs at least 11 points per bin for reliable results (specially for the error estimation; see Alexander 1997) a linear interpolation of the polarimetric data points has been performed. We must mention that due to the confusion from nearby stars and the diffuse background emission the accuracy of measuring polarimetric parameters is related to the brightness of Sgr A*. This means that the most reliable polarimetric data are measured when strong flares happen, specially when the source is in its brightest state.

In order to test whether a random red noise model can produce the same correlation patterns we need to simulate red noise light curves including polarimetric data. For this purpose, we have simulated random $E$ vectors for four perpendicular directions:

$\boldsymbol{E}_{\theta}=E_{\theta} \mathrm{e}^{-\mathrm{i} \phi t} \hat{\boldsymbol{\theta}}$

where $\theta=0^{\circ} ; 45^{\circ} ; 90^{\circ} ; 135^{\circ} ; \hat{\boldsymbol{\theta}}$ is the corresponding normal vector, $\phi$ is the phase and $E_{\theta}$ is a random value following the algorithm by Trimmer \& König (1995). Following the detection of the signal including the cross-talk from non-orthogonal neighboring channels, the polarized flux in each channel is then produced by using the Mueller matrix formalism. The resultant four different channels $\left(F_{0}, F_{45}, F_{90}\right.$ and $\left.F_{135}\right)$ all show the same power law index (Figs. 9-11a). Using

$$
\begin{aligned}
F & =F_{0}+F_{90} \\
Q & =F_{0}-F_{90} \\
U & =F_{45}-F_{135} \\
\chi & =\frac{1}{2} \arctan \left(\frac{U}{Q}\right) \\
\Pi_{\mathrm{L}} & =\frac{\sqrt{Q^{2}+U^{2}}}{F}
\end{aligned}
$$

(where $F$ is the total flux, $Q$ and $U$ are the Stokes parameters, $\chi$ is the polarization angle and $\Pi_{\mathrm{L}}$ is the degree of linear polarization) the flux and polarization light curves for each set can be derived. Figures 9-11 show examples of such light curves with three different PSD slopes: $\omega=0$ (white noise), $\omega=-1$ (flicker noise) and $\omega=-2$ (red noise).

As one can see in Fig. 11 for $\omega=-2$ some random correlations between the changes in the total flux and the polarimetric 
M. Zamaninasab et al.: NIR flares of Sgr A*
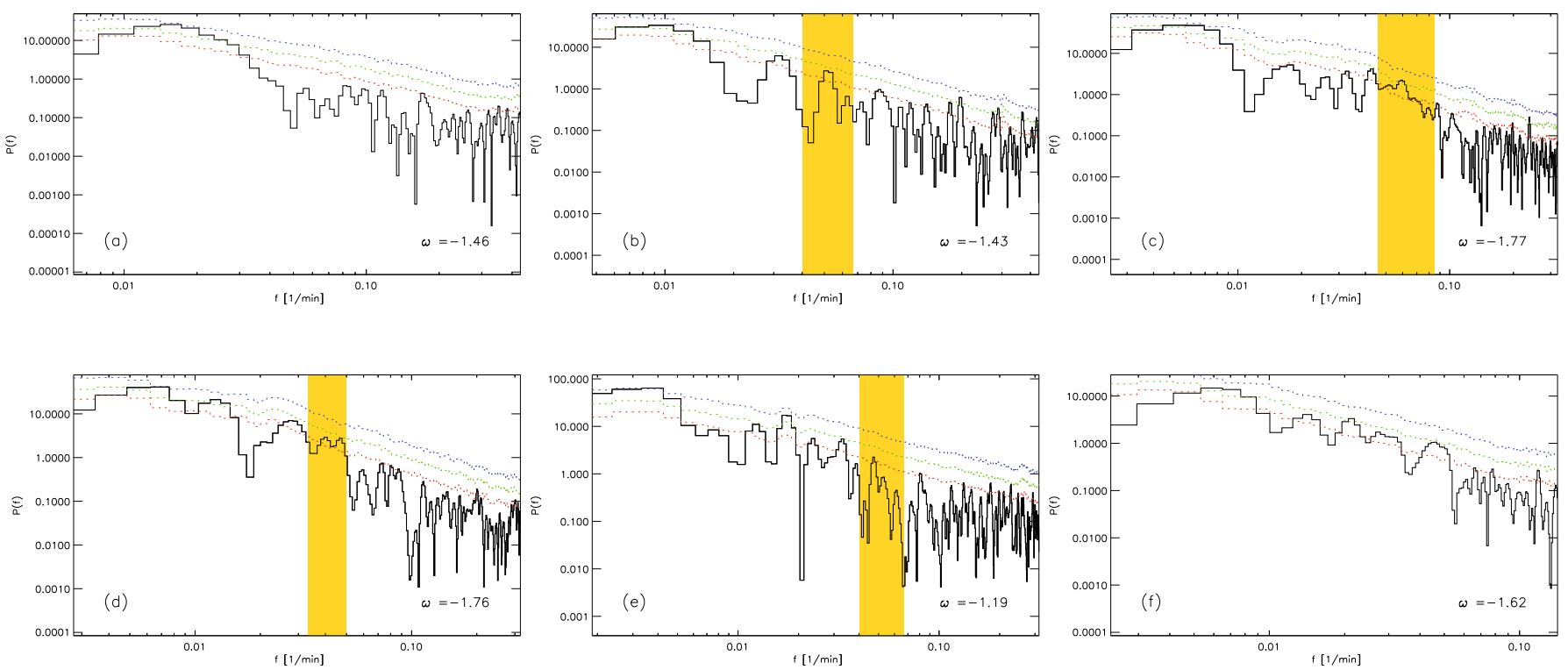

Fig. 5. Lomb-Scargle periodograms of the flux light curves for 13 june 2004 a); 30 july 2005 b); 1 june 2006 c); 15 may 2007 d); 17 may 2007 e) and 28 may 2008 f). The dashed lines show the median (red) and, $68.3 \%$ (green) and $99.8 \%$ (blue) thresholds derived from the $10^{4}$ red noise simulated light curves. The highlighted boxes show the band of frequencies that have corresponding ZDCF peaks.

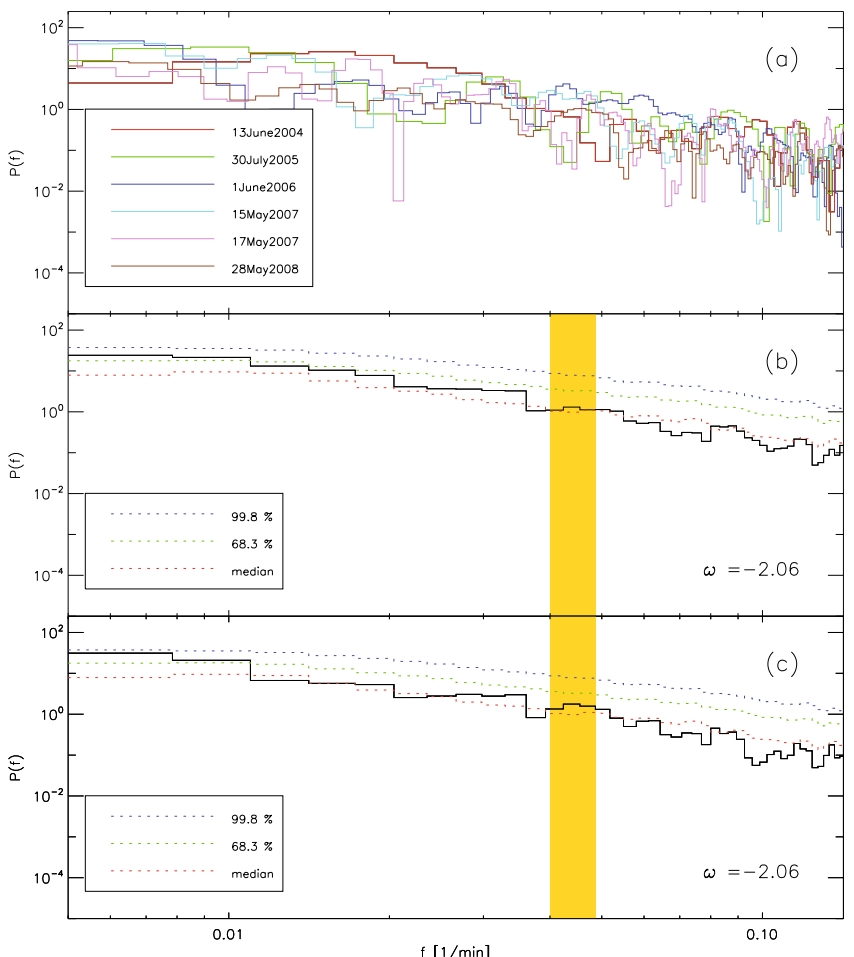

Fig. 6. Lomb-Scargle periodograms of the flux light curves of our sample overplotted in the same plot a). Averaged result of all periodograms b) and excluding the 13 june 2004 flare c). Highlighted box shows the expected $20 \pm 5$ min periodicity band.

parameters can occur. In order to see whether the observed correlations in our sample are just the same random coincidences or if they show signs of a more subtle process, we have simulated $10^{4}$ artificial light curves for each value of $\omega$ as derived from the average PSD of observations. Then for each set of light curves the correlation function between flux and polarization angle (degree) has been calculated. Dashed lines in Fig. 8 show the median, $68.3 \%$ and $99.8 \%$ false alarm values. One can see that some

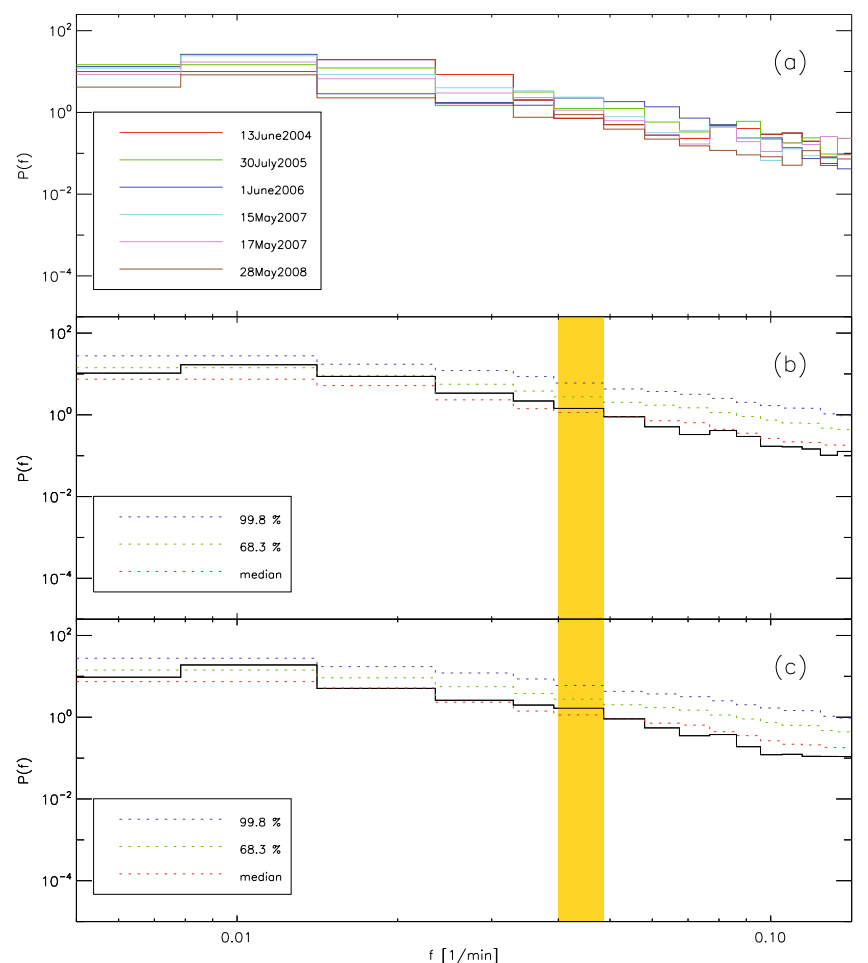

Fig. 7. Same as Fig. 6 but averaged for a band of frequencies with the same size as the highlighted region.

of the observed correlations are above $99.8 \%$ significance level. Even though most of the mentioned cross-correlation peaks happen around the same value (around zero time-lag) not all of them are exactly in the same $\tau$. To examine how probable it is that a strong deviation from the average in the red noise simulation repeatedly happens in a specific window we have calculated the probability that the mentioned cross correlations show significant peaks (above 99.8\%) in a window of a size $\delta$ (see Fig. 12b).

Figure 13 shows this probability as derived for the simulated light curves (solid line) and observations (circles and triangles). 

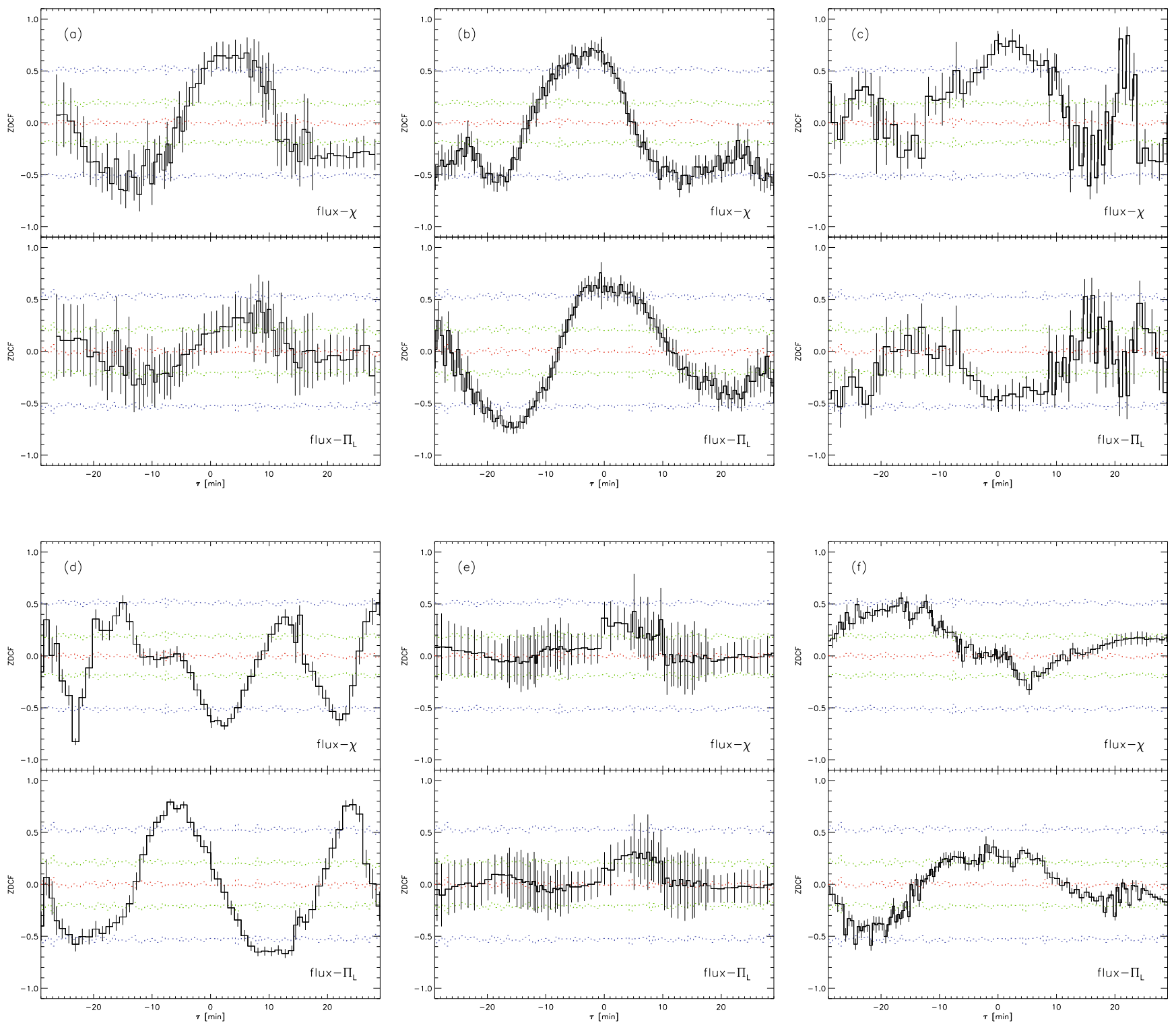

Fig. 8. Cross-correlation between the flux and polarization angle (degree) light curves of Fig. 2 (13 june 2004 a); 30 july 2005 b); 1 june 2006 c); 15 may 2007 d); 17 may 2007 e) and 28 may 2008 f)). In each panel, top (bottom) shows the correlation between the flux and the polarization angle (degree of linear polarization). Dashed lines indicate the position of the median (red), 68.3\% (green) and 99.8\% (blue) significance levels derived from $10^{4}$ simulated red noise light curves.

This analysis shows that it is very unlikely to observe a correlation between total flux and polarimetric parameters approximately at the same time lags (small $\delta$ ), while our observations show that a strong correlations exist in the light curves of Sgr A* and that they also repeat themselves for approximately the same time lag. We must note here that the value derived for the observed probability in Fig. 13 (circles and triangles) are derived from a sample of only six sets of light curves. In order to make a more reliable statistical analysis, more NIR observations of $\mathrm{Sgr} \mathrm{A}^{*}$ in polarimetric mode needs to be done in the future. Furthermore, the method described here can be used in principle for polarimetric observations of other sources showing the same variability; which may help in understanding the general underlying physical process causing this kind of behavior.

Without GR effects being taken into account, the physical models which have been already proposed to simulate the observed red-noise light curves of AGNs (Lyubarskii 1997; Armitage \& Reynolds 2003; Vaughan et al. 2003) would have difficulties in reproducing this type of correlation between the behavior of polarimetric parameters and the total flux. As a result, the observed correlation between changes in flux and the polarimetric data suggests a way to distinguish between the possible physical processes responsible for the overall red-noise behavior. A semi-analytical study by Pecháček et al. (2008) showed that a signal generated by an ensemble of spots randomly created on the accretion disk surface can produce red noise signals with PSD slopes of the order of -2 . In their simulations the spot generation is governed by Poisson or Hawkes processes. In combination with our observations of Sgr A* the spotted disk scenario is a possible explanation for this commonly observed red noise behavior, while some exceptionally luminous events can show their signature in polarized light. This could point out to the transient occurrence of QPOs which may appear repeatedly during the bright flares, which seems to be a rather natural possibility. This will be discussed in the next sections in more detail. 

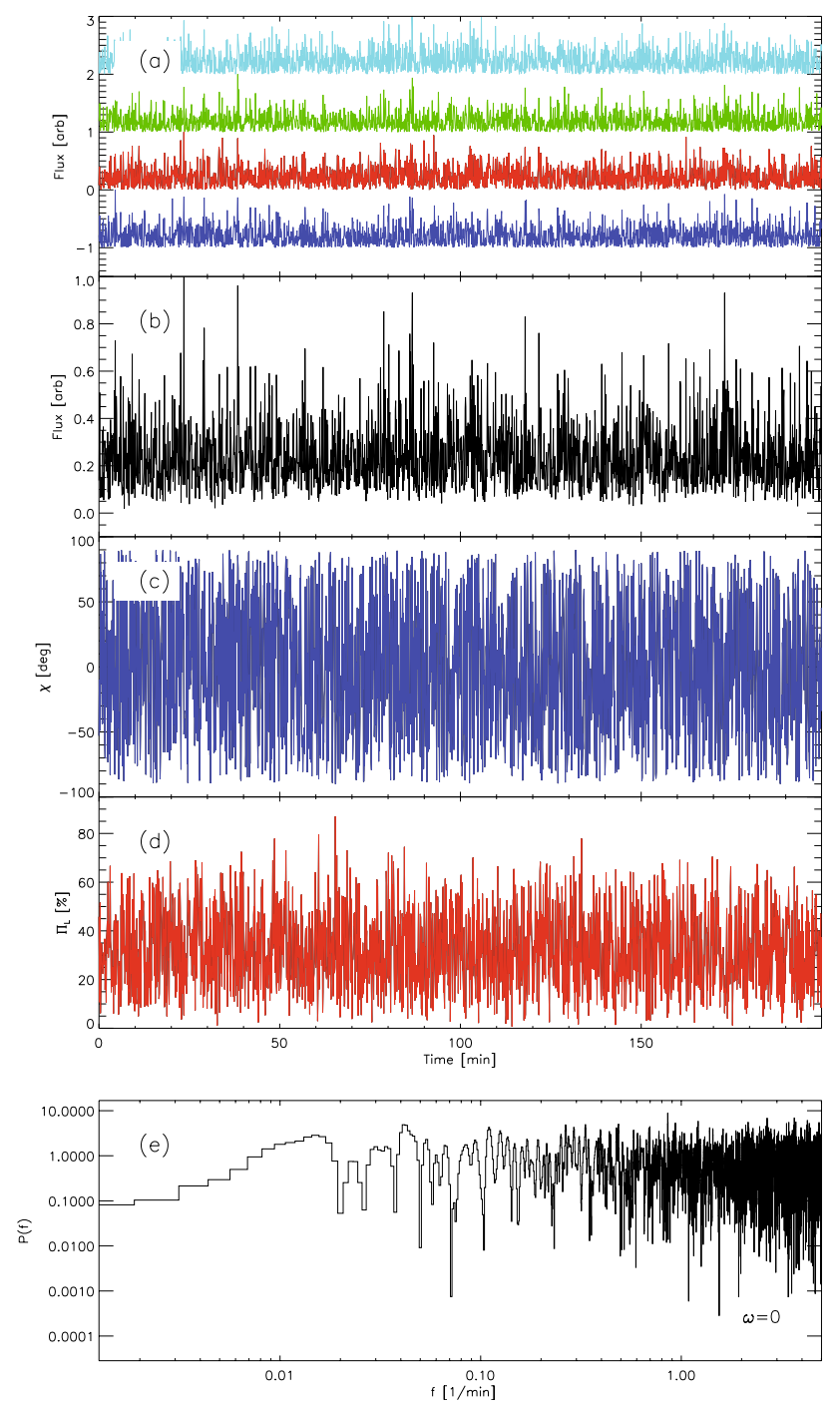

Fig. 9. a) Simulated light curves of four different polarimetric channels all showing white noise behavior $(\omega=0)$. b) Total flux. c) Angle of polarization. d) Degree of polarization. e) Lomb-Scargle periodograms of the total flux.

\section{Modeling}

In this section, we first describe in detail our emission model, which is mainly based on synchrotron emission from accelerated electrons in the inner parts of a relativistic accretion disk. We also describe the ray-tracing method used and the predictions of the model.

\subsection{Fluctuations of the inner parts of an accretion disk: A possible description for the observed signal?}

\subsubsection{Emission model}

Since the discovery of X-ray and NIR flares from Sgr A*, several flaring regions theories tried to describe the physics behind them, varying from abrupt changes in the accretion rate of a Keplerian disk (Melia et al. 2001) to the interaction of the accretion disk with nearby stars (Nayakshin et al. 2004). Although none of these scenarios can be ruled out, there are observational evidences that give more support to some of them.

For example, as we mentioned before, the frequently observed rate of NIR flares (four to five flares/day) makes it hard
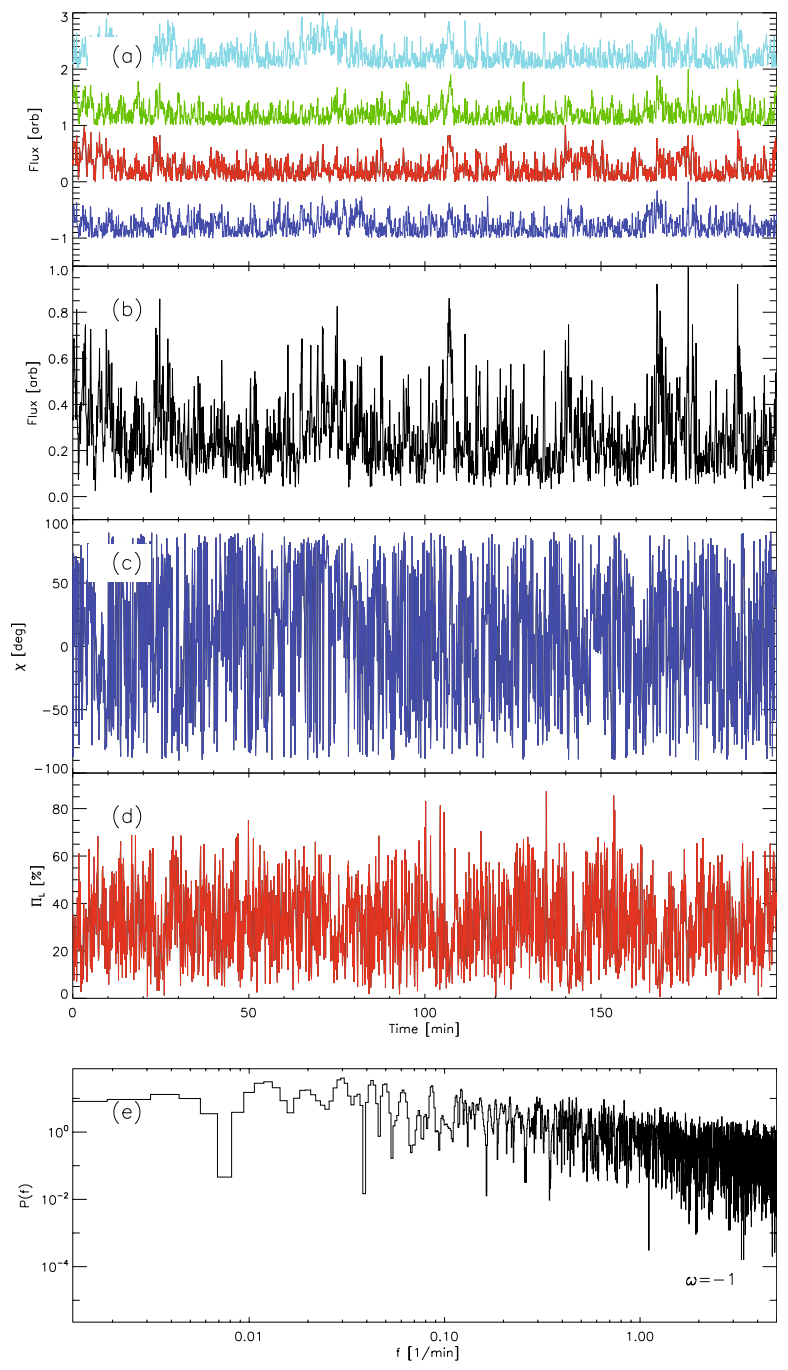

Fig. 10. Same as Fig. 9 for $\omega=-1$ (flicker noise).

for disk-star interaction or tidal capture scenarios to describe the events. Of special interest to us are the observed quasiperiodic flux modulation during the NIR and X-ray flares. The recent unambiguous discovery of (quasi-) periodicity in an active galaxy (RE J1034+396) reported by Gierliński et al. (2008) brings more support to the idea that the similarity in the behavior of black holes extends from stellar-mass black holes to supermassive ones. The most interesting scenario could be a relation to the orbital time scale of the accretion disk, with a possible connection to the plunging region which feeds the black hole through a channel of inflow or a possible clumpy infalling flow. As we describe here and in the next section, our interpretation of these variable signals (which relates flux modulations mainly to lensing and boosting effects) can open a new window to study physics in very strong gravitational regimes, very close to the event horizon of black holes.

NIR spectroscopy has shown that a power-law fit, $F_{v} \propto v^{-\alpha}$ (where $F_{v}, v$ and $\alpha$ are the flux, frequency and spectral index respectively), can describe the observed spectrum of NIR flares. Although all the observations agree with the fact that NIR flares show a soft spectrum $(\alpha>0)$, the value of the spectral index is still not well determined. The first NIR spectroscopy observations in July 2004 (Eckart et al. 2004) proposed the $\alpha$ value to be $\sim 0.8-1.3$ during the peak of the flare. In 2006, Gillessen et al. (2006) observed a correlation between flux and spectral 

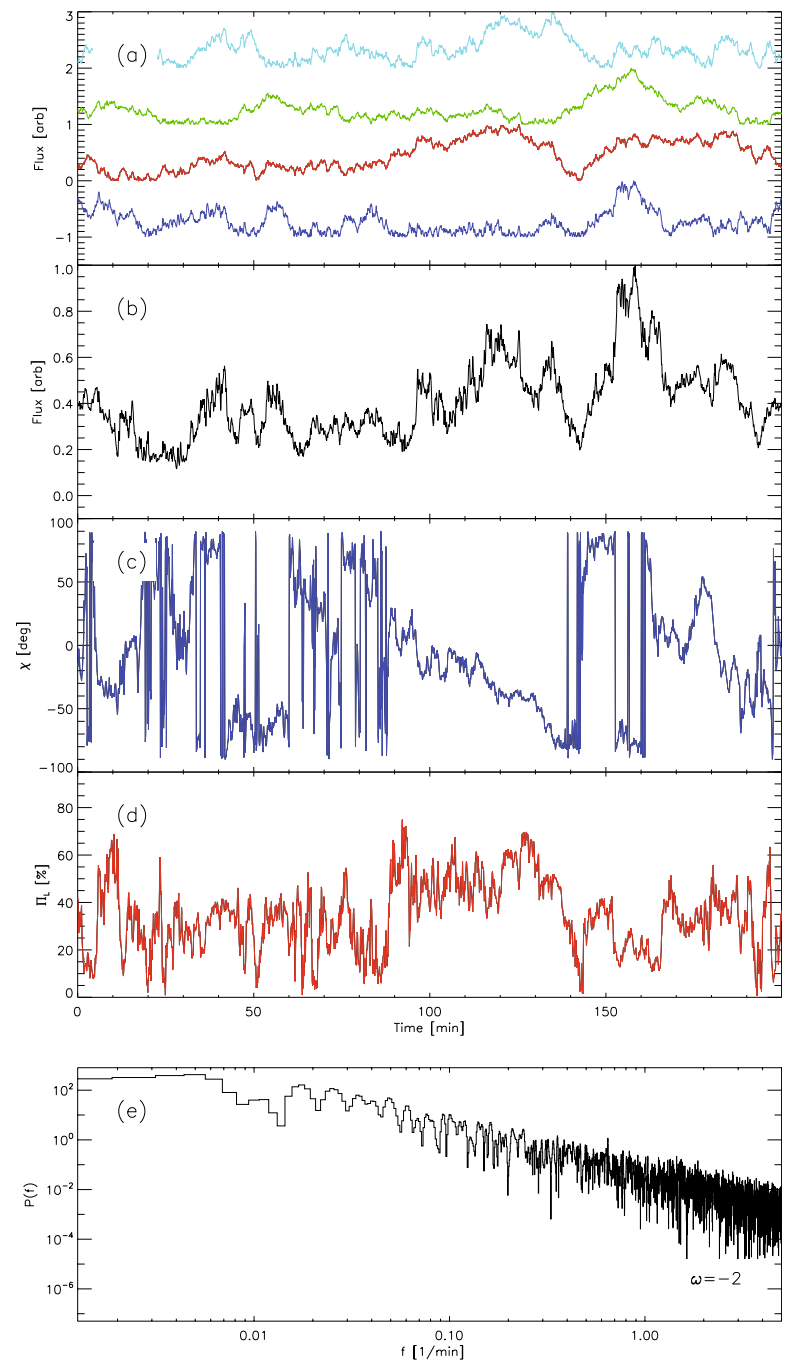

Fig. 11. Same as Fig. 9 for $\omega=-2$ (red noise).

index in their observations. However, recent observations by Hornstein et al. (2007) are consistent with a constant spectral index, $\alpha=0.6 \pm 0.2$.

The actual value of the spectral index shows its importance in the modeling of the physical process responsible for the flaring emission. Some current models (Melia et al. 2001; Liu et al. 2006; Yuan et al. 2007), predict that during flares a fraction of electrons near the event horizon of the black hole are accelerated. This can be described in the simplest form by a power law distribution in the energy of radiating electrons, $N(\gamma)=N_{0} \gamma^{-p}$ where $N(\gamma), N_{0}, \gamma$ and $p$ are the energy distribution function of electrons, normalizing constant, Lorentz factor of the electrons and the energy spectral index respectively. For high values of $\alpha$ one will need a sharp cut-off to the energy spectrum of electrons $\left(\gamma_{\mathrm{c}}\right)$, while a lower value of $\alpha(\alpha \sim 0.6)$ allows for a relatively milder distribution in the energy of electrons. Liu et al. (2006) have shown that simultaneous NIR and X-ray spectral measurements can constrain the parameters of the emitting region well.

Before describing the details of our simulations, here we discuss how the existing observations limit the possible range of free parameters. Observationally it is proven that in the Sgr A* spectrum a turn-over frequency in the sub-millimeter to NIR range exists. By using the turn-over frequency relation, $v_{\mathrm{c}}=$ $2.8 \times 10^{6} B \gamma_{\mathrm{c}}^{2} \mathrm{GHz}$, one can put an upper limit on $\gamma_{\mathrm{c}}^{2} B$, where $B$ is the magnetic field strength in Gauss. Here we have used
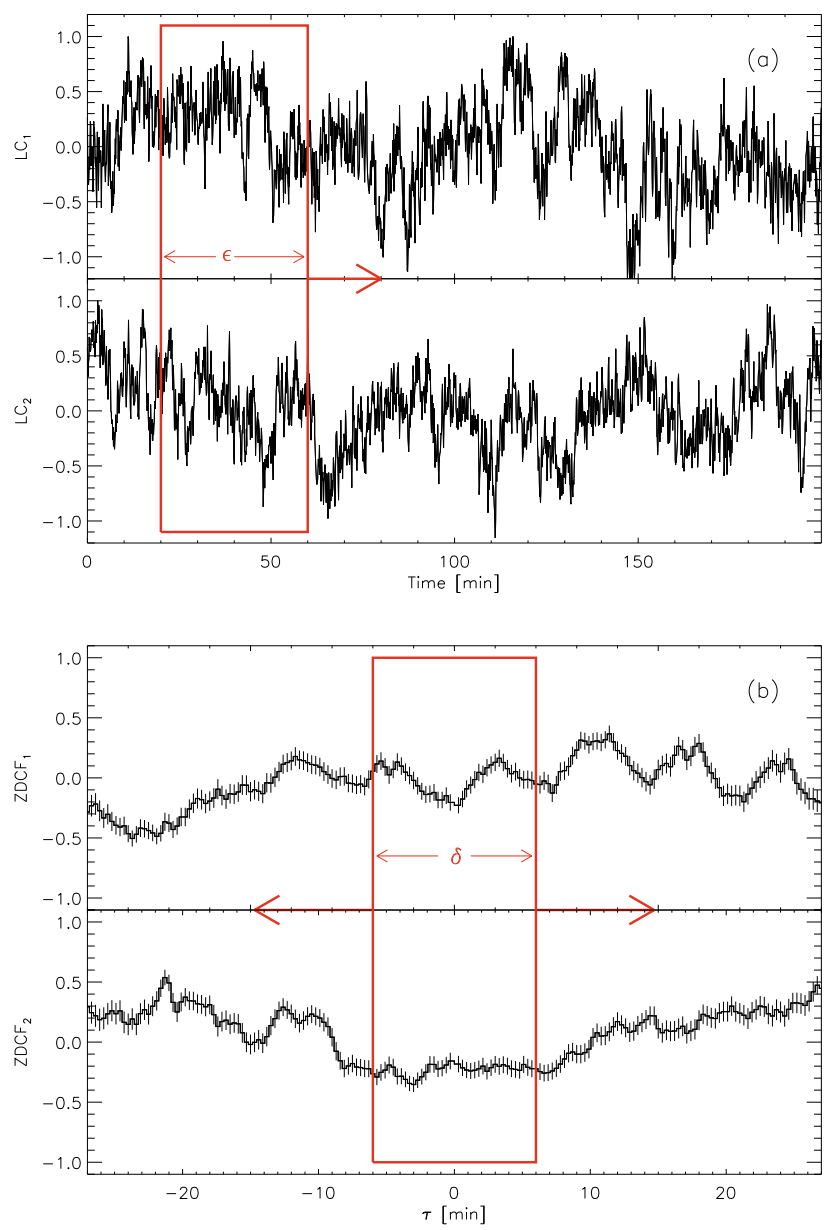

Fig. 12. a) Sketch showing how the cross-correlations of Fig. 8 have been derived. A moving window of the size $\epsilon$ scans the flux and polarization angle (degree) light curves. The final cross-correlation is the average of all windows. b) Sketch showing how the probability function in Fig. 13 has been derived. The algorithm finds the number of events in which two ZDCFs show at least one point over $3 \sigma$ threshold of red noise correlation in a window the size of $\delta$. By repeating the same procedure for $10^{4}$ cross-correlation of red noise light curves the probability that a significant correlation is expected to happen in that window is derived.

$\gamma_{\mathrm{c}}=100$ and $B=60 \mathrm{G}$ which give the best fit to the NIR/X-ray models that already exist (Liu et al. 2006; Eckart et al. 2008a).

In our simulations we first considered a scenario in which the main flare is caused by a local perturbation of intensity close to the marginally stable orbit (via magnetic reconnections, stochastic acceleration of electrons due to MHD waves, magneto rotational instabilities (MRI) etc.). These instabilities spread out and produce a temporary bright torus around the black hole. In this scenario, the mentioned variabilities are mainly due to relativistic flux modulations caused by the presence of an azimuthal asymmetry in the torus.

Simulations are dealing with two important velocities: radial $\left(v_{\mathrm{r}}\right)$ and azimuthal $\left(v_{\phi}\right)$. The radial velocity can be parameterized in the following way: $v_{\mathrm{r}} \sim\left(\frac{4 \beta_{\mathrm{P}} \beta_{v}}{9}\right)\left(\frac{G M}{r}\right)^{\frac{1}{2}}$ which depends on the ratio of the stress to the magnetic field energy density, $\beta_{v}$, and the ratio of the magnetic energy density to the thermal pressure, $\beta_{\mathrm{P}}$ (Melia 2007). The use of the typical values of $\beta_{\mathrm{P}}$ and $\beta_{v}$ from MHD simulations give us an estimation $\left(\beta_{\mathrm{P}} \beta_{v} \sim 0.05\right)$. This leads to a radial velocity of the order of $0.1\left(\frac{r_{\mathrm{g}}}{\mathrm{min}}\right)$. For the azimuthal velocity, we assumed that above the marginally stable 


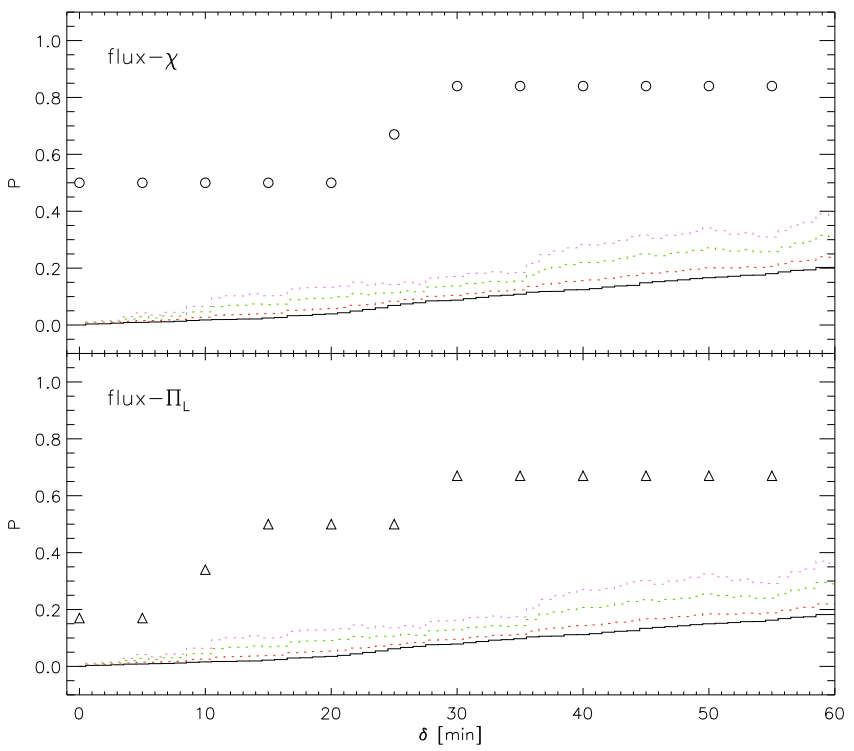

Fig. 13. Top: Probability that two sets of $10^{4}$ simulated red noise light curves show significant correlation between total flux and polarization angle in a window of the size of $\delta$ minutes (solid line). Bottom: Same as top panel for the correlation between flux and polarization degree. Dotted lines show $1 \sigma$ (red), $3 \sigma$ (green) and $5 \sigma$ (violet) levels of confidence for the probability function, calculated by repeating the whole analysis 100 times. The circles and triangles indicate the probabilities derived from our sample of NIR light curves (see Figs. 2 and 8).

orbit the plasma is in a Keplerian orbit, $v_{\phi}=\left(\frac{r^{2}-2 a \sqrt{r}+a^{2}}{\sqrt{\Delta}\left(r^{3 / 2}+a\right)}\right)$ where $\Delta=r^{2}-2 r-a^{2}$, and inside the plunging region the matter experiences free fall with the same angular momentum as at the marginally stable orbit.

Furthermore, two important time scales are at work: heating and cooling time scales. The heating time scale strongly depends on the physical processes which act as the engine of the whole event (MHD instabilities, magnetic reconnections etc.), and one can just put an observational constraint on that according to the averaged observed rise time of the events $\left(t_{\text {rise }}^{-} \sim 40 \mathrm{~min}\right)$. Cooling time is mainly controlled by the Keplerian shearing and synchrotron loss time, $t_{\text {syn }}=5 \times 10^{5} B^{-\frac{3}{2}} v^{-\frac{1}{2}}$ min, where $B$ must be set in Gauss and $v$ is in $\mathrm{GHz}$.

\subsection{Hot spot model and fluctuations of the inner parts of the accretion disk}

Since all these physical processes happen very close to the black hole and in a very strong gravitational regime, we must take into account the effects of curved space-time. To simulate the changes in paths and polarization properties of photons from the emitting electrons to the observer at infinity, we have used the KY ray-tracing code (Karas et al. 1992; Dovčiak et al. 2004). KY is able to calculate all the effects of GR, like light bending and changes in the emission angle, changes in the polarization angle of photons, gravitational lensing and redshift, Doppler boosting (since matter inside the accretion disk is in orbit) and frame dragging (in case of Kerr black holes) in a thin disk approximation. In the geometrical optics approximation, photons follow null geodesics, and their propagation is not affected by spin-spin interaction with a rotating BH (Mashoon 1973). This means that wave fronts do not depend on the photon polarization, and so the ray tracing through the curved space-time is adequate to determine the observed signals. Since our analysis is focused on high

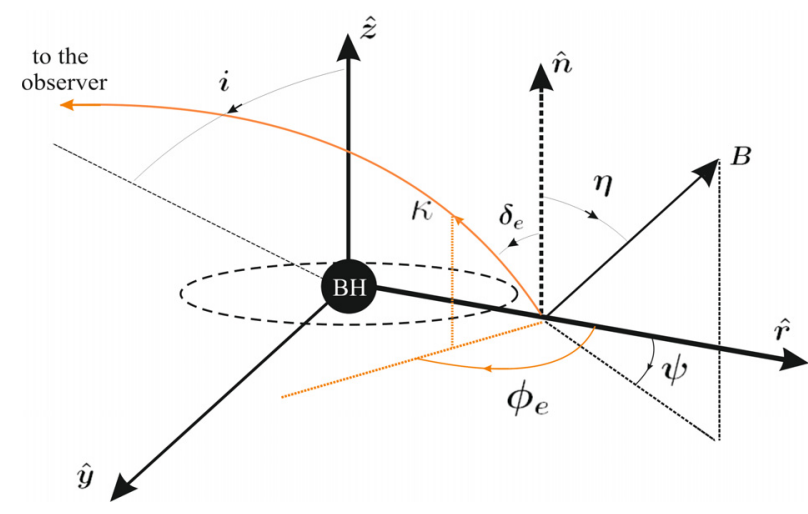

Fig. 14. The geometry we considered in our emission model. The accretion disk around the black hole lies on the $\hat{\boldsymbol{y}}-\hat{\boldsymbol{r}}$ plane and $\hat{\boldsymbol{n}}$ is the unit normal vector of the disk. The direction of the magnetic field lines (B) corresponding to the disk frame is defined by two angles $\eta$ and $\psi$. $\kappa$ represents the momentum of the emitted photon and its direction determined by a set of angles $\left[\delta_{\mathrm{e}}, \phi_{\mathrm{e}}\right]$. The distant observer is looking into the system along a line of sight inclined by a certain angle $i$.

frequency regimes, we have mainly ignored radiative transfer effects.

To make KY work, we must initialize the properties of radiated photons at each point of the emitting region. The straightforward way is to define the intrinsic emission of each point in the context of the Stokes parameters. For the flux densities (mJy) and source sizes ( $\mu$ as) of $\operatorname{Sgr} \mathrm{A}^{*}$, optically thick synchrotron emission in the NIR can safely be disregarded (see the discussion in Eckart et al. 2009). Since in this report we focus only on the modeling of the NIR flares, it is sufficient to pick up a model for the energy distribution of non-thermal electrons, radiating in an optically thin regime:

$N(\gamma)=\left\{\begin{array}{cc}N_{0} \gamma^{-p} & \gamma \leq \gamma_{\mathrm{c}} \\ 0 & \gamma>\gamma_{\mathrm{c}}\end{array}\right.$

which leads to the formulae for polarized emission:

$$
\begin{aligned}
& I_{v} \propto n\left(B \sin \theta_{\mathrm{e}}\right)^{\left(\frac{p+1}{2}\right)} v^{-\left(\frac{p-1}{2}\right)} \\
& Q_{v}=\Pi_{\mathrm{L}} \cos \left(2 \chi_{\mathrm{e}}\right) I_{v} \\
& U_{v}=\Pi_{\mathrm{L}} \sin \left(2 \chi_{\mathrm{e}}\right) I_{v} \\
& V_{v}=\Pi_{\mathrm{C}} I_{v}
\end{aligned}
$$

where $n, \chi_{\mathrm{e}}, \Pi_{\mathrm{L}}$ and $\Pi_{\mathrm{C}}$ are the number density of the electrons, the angle between a reference direction and the plane of an observer co-moving with the disk frame and degree of linear and circular polarization, respectively. Throughout this paper we assume that in the NIR regime the light is not circularly polarized $\left(\Pi_{\mathrm{C}}=0\right) . I_{v}, Q_{v}, U_{v}$, and $V_{v}$ represent the Stokes parameters. $\theta_{\mathrm{e}}$ is the angle between the direction of the magnetic field and the direction toward the co-moving observer

$\theta_{\mathrm{e}}=\theta_{\mathrm{e}}\left(\eta, \psi, \phi_{\mathrm{e}}, \delta_{\mathrm{e}}\right)=\arccos \left(\sqrt{\frac{\left(\mathcal{B}^{\alpha} \kappa_{\mathrm{e} \alpha}\right)^{2}}{\left(\kappa_{\mathrm{e}}^{\beta} \kappa_{\mathrm{e} \beta}\right)\left(\mathcal{B}^{\gamma} \mathcal{B}_{\gamma}\right)}}\right)$

where we have picked the disk co-moving frame as the reference, so that the normal to the disk, $\hat{n}$, coincides with the $\hat{z}$ direction. Geometrical orientation of the dominant component of the global magnetic field vector $(\mathcal{B})$ in this frame can be defined with a set of angles $[\eta, \psi](0 \leq \eta \leq \pi, 0 \leq \psi \leq 2 \pi$, see Fig. 14). 
$\delta_{\mathrm{e}}$ is the angle between the direction of the photon momentum $(\kappa)$ and the normal to the disk $(\hat{\boldsymbol{n}})$ :

$\delta_{\mathrm{e}}=\arccos \left(-\frac{\kappa_{\mathrm{e} \alpha} n^{\alpha}}{\kappa_{\mathrm{e} \beta} \nu^{\beta}}\right)$

where $v$ is the four-velocity of matter in the disc. $\phi_{\mathrm{e}}$ is the azimuthal emission angle which is defined as the angle between the projection of the three momentum of the emitted photon into the equatorial plane and the radial tetrad vector:

$\phi_{\mathrm{e}}=\arctan \left(\frac{\kappa_{\mathrm{e}}^{\alpha} \hat{\boldsymbol{e}}_{(\phi) \alpha}}{\kappa_{\mathrm{e}}^{\mu} \hat{\boldsymbol{e}}_{(r) \mu}}\right)$.

Having all the needed information at hand, by fixing free parameters like the spatial density distribution of the emitting electrons, magnetic field strength, flux spectral index, and the global configuration of the magnetic field, one can simulate the expected images that a distant observer will measure for different possible inclinations with respect to the black hole/accretion disk system. Here we will show how compact azimuthal anomalies inside a uniform density distribution of the emitting plasma can reproduce the observed behavior of $\mathrm{Sgr} \mathrm{A}^{*}$ in high frequency regimes.

The synchrotron cooling time at NIR frequencies is only of the order of a few minutes, which is significantly shorter than the time scale of the observed flares. One way that a spot can survive long enough to be responsible for the observed $\sim 4-5$ time flux modulations is that a SSC mechanism up-scatters the sub-mm seed photons to the NIR and X-ray frequencies (Eckart et al. 2006a-c, 2008a). The other possibility is that the heating time of the orbiting NIR component is related to the rise time of the main flare, $\tau \propto t_{\text {rise }}$ where the emissivity profile of the emitting component follows $F\left(t ; t_{0}, \tau\right)=F_{0} \exp \left(-\left(t-t_{0}\right)^{2} / 2 \tau^{2}\right)$.

The above discussion demonstrates that it is essential to consider the gravitational shearing time scale as a variable in the simulations. We have implemented this effect in our modeling by introducing a dimensionless characteristic shearing time scale:

$\tau_{\mathrm{sh}}=\frac{T\left(r_{\mathrm{spot}}\right)}{T\left(r_{\mathrm{spot}}+r_{0}\right)-T\left(r_{\mathrm{spot}}-r_{0}\right)}$

where for the initial spatial distribution of the relativistic electrons we have used a spherical Gaussian distribution with its maximum being located at the radius $r=r_{\text {spot }}$ with $F W H M$ of $r_{0}=1 r_{\mathrm{s}}$. In our simulations, $\tau_{\mathrm{sh}}$ varies between $\infty$ and 0.8 . $\tau_{\mathrm{sh}}=\infty$ corresponds to the situation in which the spot preserves its shape for a long time. The mechanism that stabilizes the spot is not known, although several possibilities have been proposed and explored in the literature. In particular, the existence of persistent vortices on accretion disks has been explored (Abramowicz et al. 1992; Adams \& Watkins 1995). However, our modeling suggests that shearing effects are indeed important and can be well represented within the multi-component scheme or in the spiral pattern scheme generalizing the original spot scenario. The $\tau_{\mathrm{sh}}=0.8$ corresponds to a pure Keplerian shearing for a spot located at the marginally stable orbit of an extreme spinning black hole $(a=1)$. One must note that MHD simulations are unable to produce hot spots with long life-times comparable to the observed flare time scales (Hawley et al. 2001; Krolik et al. 2002). Schnittman et al. (2006) used a model for the creation and annihilation of spots with short life times, distributed by random phase within a belt close to the marginally stable orbit. They show that their model can resemble the observed quasiperiodicity in the X-ray light curves. Eckart et al. (2008a) successfully modeled the simultaneous NIR/X-ray flares of Sgr A* by following the same basic idea of a multi-component model. As mentioned in Sect. 2, following the results of Pecháček et al. (2008) these kinds of multi-component models are able to reproduce the red noise behavior of PSDs while some bright individuals can show their signatures in polarized light (see Fig. 25 and also discussion in Eckart et al. 2008a).

\subsection{Results of the modeling}

Figure 15 shows how a hot spot is created and evolves in time for three different values of $\tau_{\mathrm{sh}}$. A comparison between the rows shows how pure Keplerian shearing disrupts the initial shape of the spot and produces an elongated spiral shape. Figure 16 shows the apparent images of a spot with a mild shearing environment $\left(\tau_{\mathrm{sh}}=2.0\right)$ for three different inclination angles. When we look face-on at the event $\left(i \simeq 0^{\circ}\right)$, there are no modulations by the relativistic effects. For higher inclination angles ( $i=30^{\circ}$ and $i=60^{\circ}$ ) lensing and boosting effects play major roles in the observed flux modulations. Specially for high inclinations one can see how an Einstein arc develops when the spot passes behind the black hole and how photons coming from the accretion disk are blue-shifted on the left hand side of the image according to Doppler boosting. This set-up allows us to simulate light curves for a wide range of possible free parameters, mainly by covering the range of all possible inclinations and spins of the black hole. Figure 17 shows examples of light curves for different values of inclination and shearing time scale.

\subsubsection{Pattern recognition analysis: signatures of lensing effects}

Figure 18 shows the typical magnification of flux, polarization angle and degree of an orbiting spot emission as a function of time. Here we showed the spots located at different distances from the black hole. These plots indicate the typical behavior of light curves when the strong gravitational regime is prominent and the strong lensing and boosting is happening. As one can see, the sweep in the polarization angle precedes the peak in flux magnification, while the peak of the degree of polarization follows the magnification peak (see Broderick \& Loeb 2006, for a detailed discussion). Figure 19 shows more clearly this typical behavior according to the position of the corresponding cross-correlation's peaks. It is particularly apparent that even with changing the position of the emitting source with respect to the black hole this effect remains more or less the same.

This constant behavior encouraged us to check whether or not this type of pattern is manifested in our NIR light curves of Sgr $A^{*}$. For this purpose we used a simple pattern recognition algorithm mainly via a multiplication of different cross correlation functions. Similar pattern recognition algorithms are used to identify gravitational wave signals from noisy data (Pappa et al. 2003; Goggin 2008).

We derived a final pattern recognition coefficient product by multiplying two cross correlation functions (namely $\sigma_{i}$ and $\sigma_{j}$ ) as below:

$\Sigma_{i j}=\sqrt{\left(\sigma_{i}+1\right)\left(\sigma_{j}+1\right)}-1$.

By applying this procedure for the cross correlation functions between the observed flux and the theoretical magnification light curve $\left(\sigma_{f}\right)$, cross correlation functions between observed polarization angle and the theoretical polarization angle light curve $\left(\sigma_{\chi}\right)$ and the same function for the degree of polarization $\left(\sigma_{\Pi}\right)$, we derived a final pattern recognition coefficient product $(\Sigma)$. 


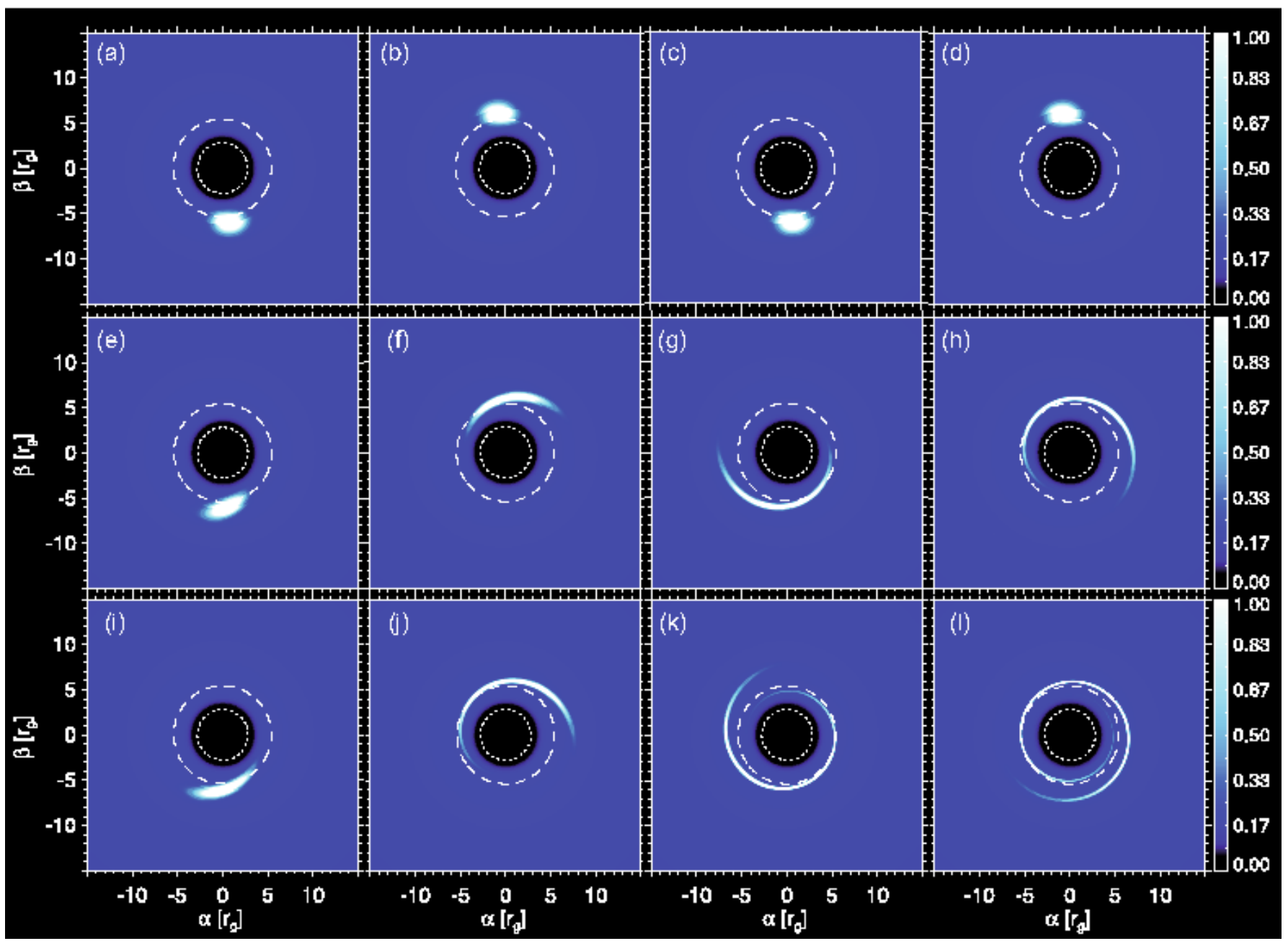

Fig. 15. Snapshots of orbiting anomalies inside the accretion disk as they appear to a distant observer looking along a line of sight inclined by $0^{\circ}$ (relative to the normal to the disk), at times after $\frac{1}{4} T, \frac{3}{4} T, \frac{5}{4} T$ and $\frac{7}{4} T$ (left to right). Each row shows how the event evolves in time for different values of the characteristic shearing time scale: $\left.\left.\left.\tau_{\mathrm{sh}}=\infty(\mathbf{a})-\mathbf{d}\right)\right), \tau_{\mathrm{sh}}=2.0(\mathbf{e})-\mathbf{h}\right)$ ) and $\left.\tau_{\mathrm{sh}}=1.0(\mathbf{i})-\mathbf{l}\right)$ ). The spin of the black hole is set to 0.5 . The dotted and dashed lines indicate the position of the event horizon and marginally stable orbit, respectively. Each row has been scaled by its maximum intensity for illustrative purposes. $\alpha$ and $\beta$ are the projections of the impact parameter of the emitted photons as the coordinates on the sky of the observer. Both coordinates are labeled in $r_{\mathrm{g}}(\simeq 5 \mu$ as $)$ units.

Figure 20 shows how this pattern recognition coefficient is defined.

Figure 21 shows the result of our pattern recognition analysis for the polarized flare events discussed in this paper. In order to estimate how significant the peaks in the $\Sigma$ function are, we have repeated the same analysis for $10^{4}$ random red noise light curves simulated with the same method mentioned in Sect. 2.2.2. In all but one case the patterns shown in Fig. 21 can be identified at the $>3 \sigma$ to $5 \sigma$ level. This shows that strong lensing patterns are significantly manifested in our sample of NIR light curves. The strong lensing pattern detected is a further indicator for the existence of a (clumpy) accretion disk around Sgr A*.

\subsubsection{A spotted accretion disk?}

Figure 22 shows a selected $100 \mathrm{~min}$ window of the resultant light curves of the flux density, polarization degree and angle for a spot with constant shape $\left(\tau_{\mathrm{sh}}=\infty\right)$, orbiting around a Kerr black hole $(a=0.5)$ close to its marginally stable orbit $\left(r=1.1 \times r_{\mathrm{mso}}\right)$. The line of sight is inclined by 60 degrees $\left(i=60^{\circ}\right.$ ). Gaussian white noise has been added to the simulated data in order to make the comparison of the periodicity and cross correlation with corresponding observational results easier. The level of the noise and the average error bars have been set from the average rms of the corresponding observed light curves. The surface brightness of the components follows a profile similar to $F\left(t ; t_{0}, \tau\right)$ with $\tau=25$. The maximum degree of polarization which can be achieved via synchrotron mechanism is around $70 \%$. Since any kind of deviation from the ideal isotropic distribution of electrons around the magnetic field lines will suppress the degree of linear polarization, we set the initial value for the radiation from the spot to be $50 \%$. We assumed that the photons originating from the non-flaring part of the accretion disk are weakly polarized $(\sim 1 \%)$, since the main population of its NIR photons have thermal origin and relativistic electrons are randomly distributed around the magnetic field lines.

Figure 23 shows the result from autocorrelation and LombScargle analysis (similar to Figs. 3 and 5), and Fig. 24 shows the results of the cross correlation analysis (similar to Fig. 8). The peaks close to the 0 min time-lag are of special interest since 


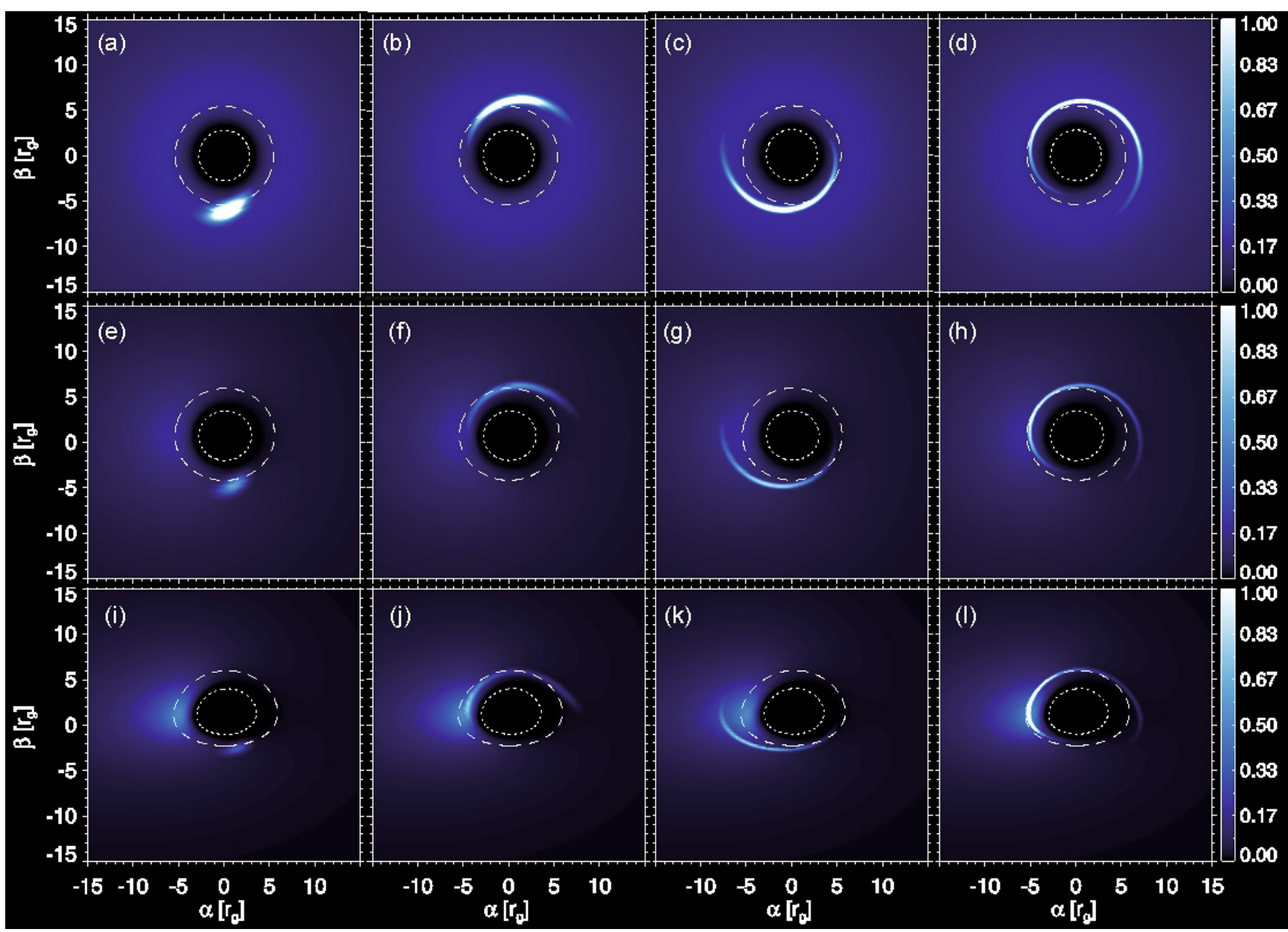

Fig. 16. Snapshots of an orbiting anomaly inside the accretion disk as it appears to a distant observer looking along a line of sight inclined by $0^{\circ}$ (a)-d)), $30^{\circ}$ (e)-h)) and $60^{\circ}$ (i)-l)) (relative to the normal to the disk) at times after $\frac{1}{4} T, \frac{3}{4} T, \frac{5}{4} T$ and $\frac{7}{4} T$ (left to right). See also the caption of Fig. 15.

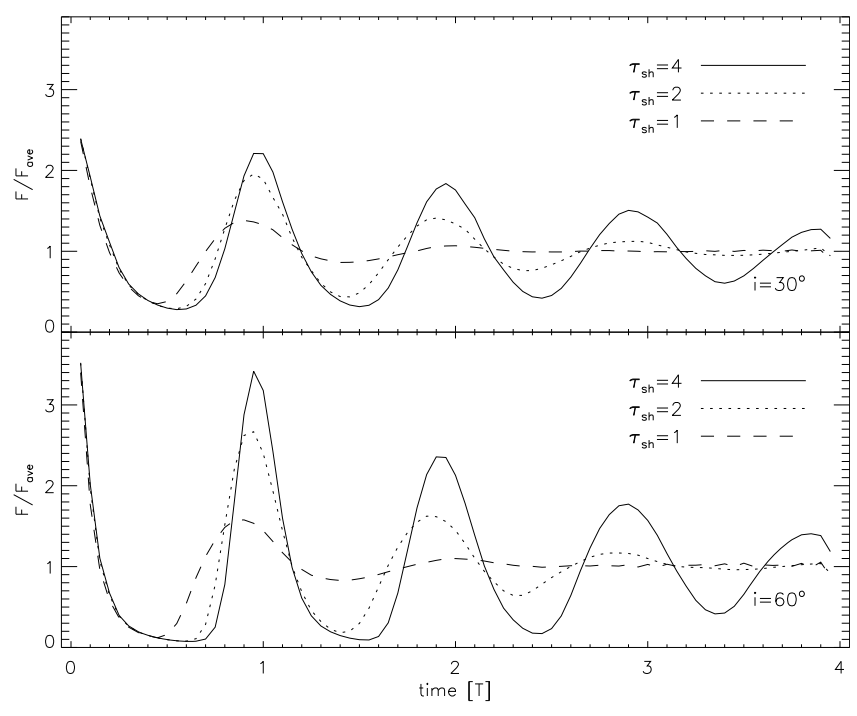

Fig. 17. Flux modulations of an evolving perturbation close to the marginally stable orbit of a Kerr black hole with spin parameter of 0.5 . The light curves show how different values of shearing parameter and inclination affects the resultant light curve.

they have the least dependency on the choice of free parameters and are mainly related to the basic idea that the flux modulations are caused by relativistic effects. Figures 23 and 24 show that an orbiting spot is able to produce the same cross correlation pattern observed in our sample, but the orbital frequency of the spot will be detected to be mainly a quasi-periodical signal. The corresponding correlation functions of the 30 july 2005 observation are over-plotted in Fig. 24 for a better comparison. The main peak close to $\tau=0$ coincides very well for both observation and simulated cross-correlations.

Furthermore, we have simulated a spotted accretion disk. In this case spots are born, evolve and finally fade away as a function of time. These anomalies are distributed in the inner part of the accretion disk in a belt between $1-2 \times r_{\text {mso }}$. Radial and azimuthal distribution of the anomalies are completely random and their distribution in time follows a simple Poisson point process (see Pecháček et al. 2008).

Figure 25 shows the snapshots of the spotted disk scenario as viewed by a distant observer from different inclination angles. The resulting magnification and polarimetric light curves are depicted in Fig. 26. White noise is added to all light curves in order to make the comparison between ZDCF and LombScargle results with the corresponding observational results easier. As can be seen in Fig. 27 the random distribution of the spots strongly suppresses the periodic signal in the Lomb-Scargle periodogram, while the cross correlation between the magnification and the changes in the polarized flux still carries a significant signal from the modulations influenced by strong gravity (Fig. 28). 


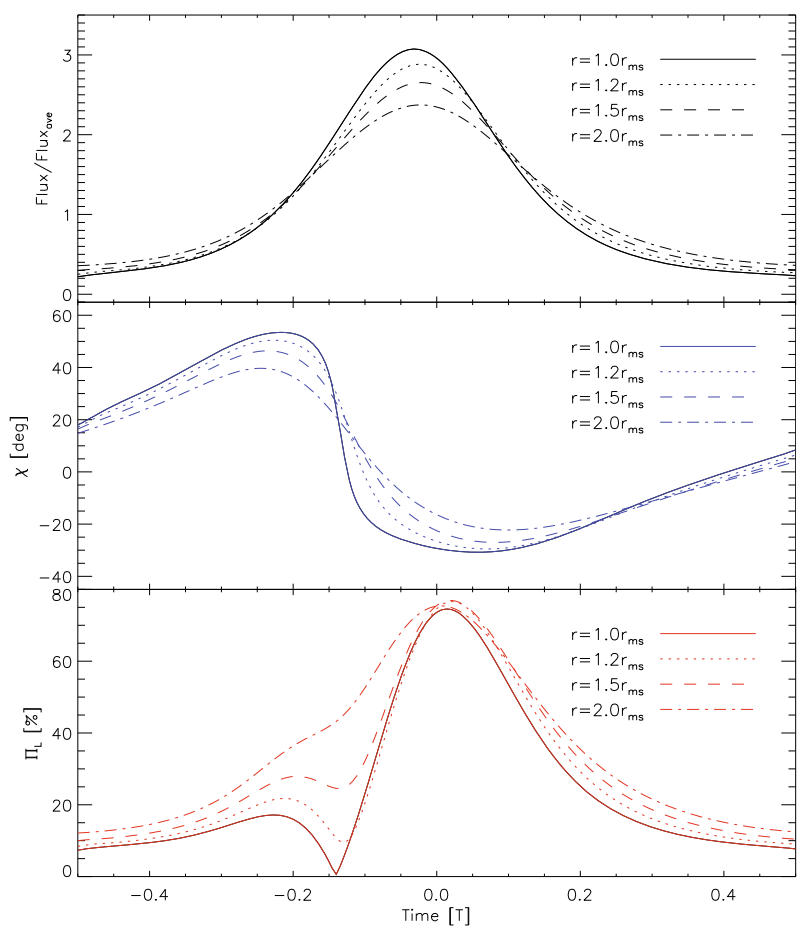

Fig. 18. Flux modulation (top), changes in polarization angle (middle) and degree (bottom) for a spot on a circular orbit at $1.0 \times r_{\mathrm{ms}}$ (solid), $1.2 \times r_{\mathrm{ms}}$ (dotted), $1.5 \times r_{\mathrm{ms}}$ (dashed), $2.0 \times r_{\mathrm{ms}}$ (dott-dashed) around a Kerr black hole with a spin parameter of 0.5 . The time unit is the orbital time scale $(T)$.

As a conclusion one can say that a general relativistic simulation of turbulences in the inner parts of an accretion disk resembles the observed behavior of Sgr A* very well.

One must note that for the simulated data, we have chosen typical values for spin and inclination (consistent with previous results by Eckart et al. 2006b and Meyer et al. 2006a,b), not trying to fit the actual data. As we can see, the data analysis and simulations show that even if there does exist a low-level activity physical process in Sgr A* which can be explained as random red-noise, the kind of observed correlations between the flux and polarization data cannot be produced via a completely random process (without taking into account relativistic effects). Actually, the similarity between Figs. 24 and 28 and what is already observed in at least three different Sgr A* flares (Figs. 5 and 8), supports the idea of orbiting matter around the galactic super-massive black hole.

\subsection{Alternative models}

As mentioned before, the strength of ZDCF's peaks in Fig. 3 seems to be correlated with the brightness of flare events. This dependency on the brightness of the flares and also the shape of the resulting ZDCFs are very similar to the expected autocorrelation if the flux variations originate from geometric "light echoes" (Bursa et al. 2007; Fukumura et al. 2009). The expected periodicities from these models are different from the ones observed here, but their very close similarity keeps this issue open to future discussions. If a more detailed modeling of random flares happening at a distance from the black hole can show a possible $20 \mathrm{~min}$ QPO, then the light curve observed on 15 and 17 may 2007 could be the first observation of second images created from separated photon "bunches" (Fukumura 2009).

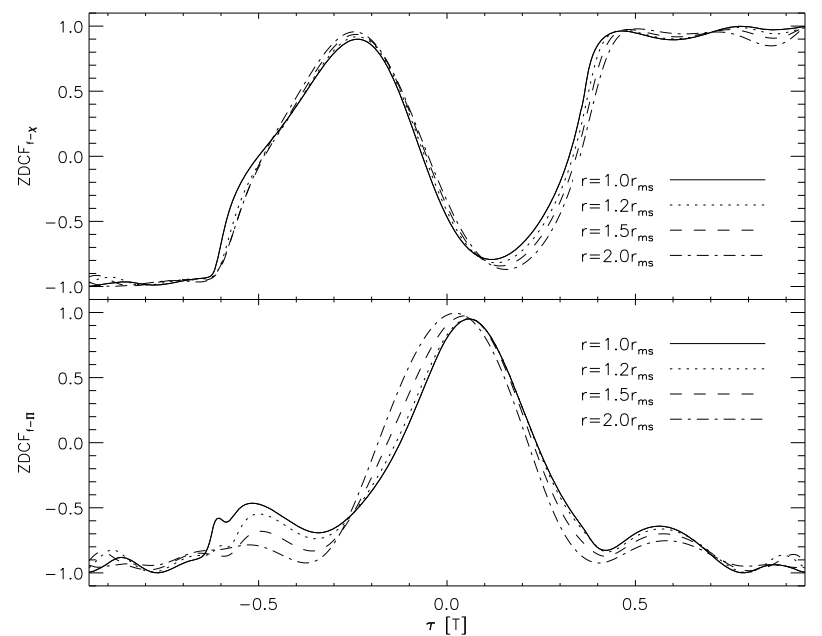

Fig. 19. Cross correlation between the changes in flux and polarization angle (top), and flux and polarization degree (bottom) for spot circular orbits at $1.0 \times r_{\mathrm{ms}}$ (solid), $1.2 \times r_{\mathrm{ms}}$ (dotted), $1.5 \times r_{\mathrm{ms}}$ (dashed), $2.0 \times r_{\mathrm{ms}}$ (dott-dashed) around a Kerr black hole with a spin parameter of 0.5 (Fig. 18). The time unit is the orbital time scale $(T)$.

Furthermore, shocks in relativistic jets can produce a correlated total flux and polarized intensity. There is a possibility that under special circumstances (e.g. special inclination, special magnetic field configuration etc.) a (episodic) relativistic outflow could produce the same correlation and pattern we have discovered in our observations.

\section{Geometry of the emitting region}

In this section we discuss the basic assumption of the existence of an azimuthal asymmetry in the accretion flow of Sgr A*. Recently, Falanga et al. (2007) and Karas et al. (2007) discussed that a global spiral pattern of disturbance, with an orbiting speed not directly associated with the underlying Keplerian velocity, can fit the observed NIR and X-ray modulations of Sgr A*. Their model has been used to fit the observed X-ray flare on 31 august 2004 (Falanga et al. 2007). Here we will show how a combination of polarimetric observations and the next generation of VLTI measurements can reveal the geometry of the emitting region of these high frequency photons.

In order to reproduce the same density profile in the inner part of the disk, we have used a spiral pattern, characterized by the emissivity function given below:

$I_{v} \propto r^{-\gamma} \sin ^{\beta}\left(\phi+\alpha \log \left(\frac{r}{r_{0}}\right)\right)$

while the power-law index, $\gamma$, describes the overall radial decrease in the emissivity, $\arctan (\alpha)$ is the pitch angle and $r_{0}$ determines the outer radius where the pattern fades away. This parametrization approximates a spiral pattern evolving in the background of a Keplerian disk. The main emission mechanism is assumed to be synchrotron radiation from relativistic electrons with the same energy distribution as Eq. (1). The shape of the spiral pattern highly depends on $\gamma, \beta, \alpha$ and $r_{0}$. We parameterize our model in such a way that it facilitates a straightforward comparison with Tagger et al. (2006). $\gamma=1, \alpha=\beta=6$ and $r_{0}=20$ give the best approximation (see Fig. 29). Figure 30 shows the changes in the flux and polarimetric quantities, as measured by a 

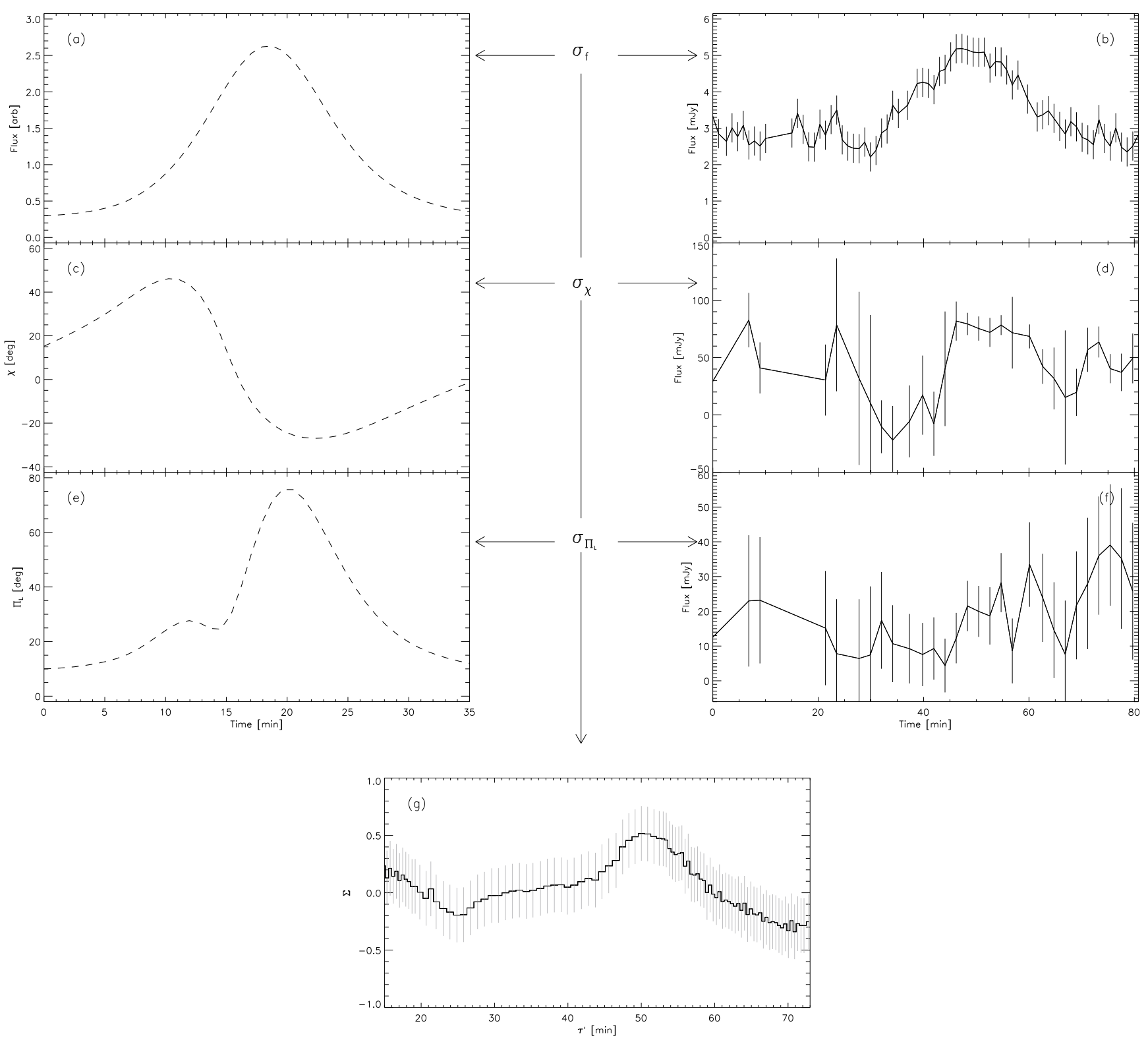

Fig. 20. A sketch showing how the pattern recognition coefficient $(\Sigma)$ is defined. $\Sigma$ is the multiplication product of the cross correlation between observed and theoretical flux $\left(\sigma_{f}\right)$, observed and theoretical polarization angle $\left(\sigma_{\chi}\right)$ and observed and theoretical polarization degree $\left(\sigma_{\Pi}\right)$ light curves following Eq. (16). Note that $\Sigma\left(\tau^{\prime}\right)(\mathrm{g})$ is defined as a function of $\tau^{\prime}=\tau+\frac{T_{\mathrm{sim}}}{2}$ (where $\tau$ is the time lag in units of minutes) in order to make it easier to match the position of its peaks with the position of the lensing events in the observations.

distant observer for different inclinations. As mentioned before (and discussed in detail by Falanga et al. 2007 and Karas et al. 2008), such a spiral pattern can produce the same typical behavior in flux modulation as that caused by an azimuthal anomaly in the accretion disk.

The main idea in our simulations is to include the polarimetric radiation transfer in curved space-time, which provides the possibility to compare the behavior of different geometrical configurations. It seems that even though both of these geometrical set-ups (spiral shape or compact spot) show the same behavior in flux, the observed polarized flux will behave significantly different. In order to reduce the effects of our specific assumptions about the emission process and magnify the signatures which result only from different geometrical structures, we have chosen a toy model for the initial polarized emission: $Q_{v}=I_{v}, U_{v}=V_{v}=0$. Figure 30 shows that the spiral pattern is unable to produce strong changes in the polarization angle, while a compact azimuthal source produces a highly variable polarization angle. Due to abberation, photons that come from different parts of the disk are polarized differently, even for Schwarzschild black holes. For the Kerr case, the rotation of the polarization vector will be added because of gravitational frame dragging. The dependency of these changes on the position of the emitted photon is depicted in Fig. 31. The top panel of Fig. 31 shows how the polarization vector of the emitted photons will be rotated due to the strong gravity of the central black hole. As one can see, there is a clear knot visible in this contour graph. If the radiating source passes through this knot, the observed polarization angle will swing dramatically (for a detailed discussion see Dovčiak et al. 2008). As the bottom panel of Fig. 31 shows, when a compact azimuthal source orbiting around the black hole is close to its marginally stable orbit, this nod will be passed. The 

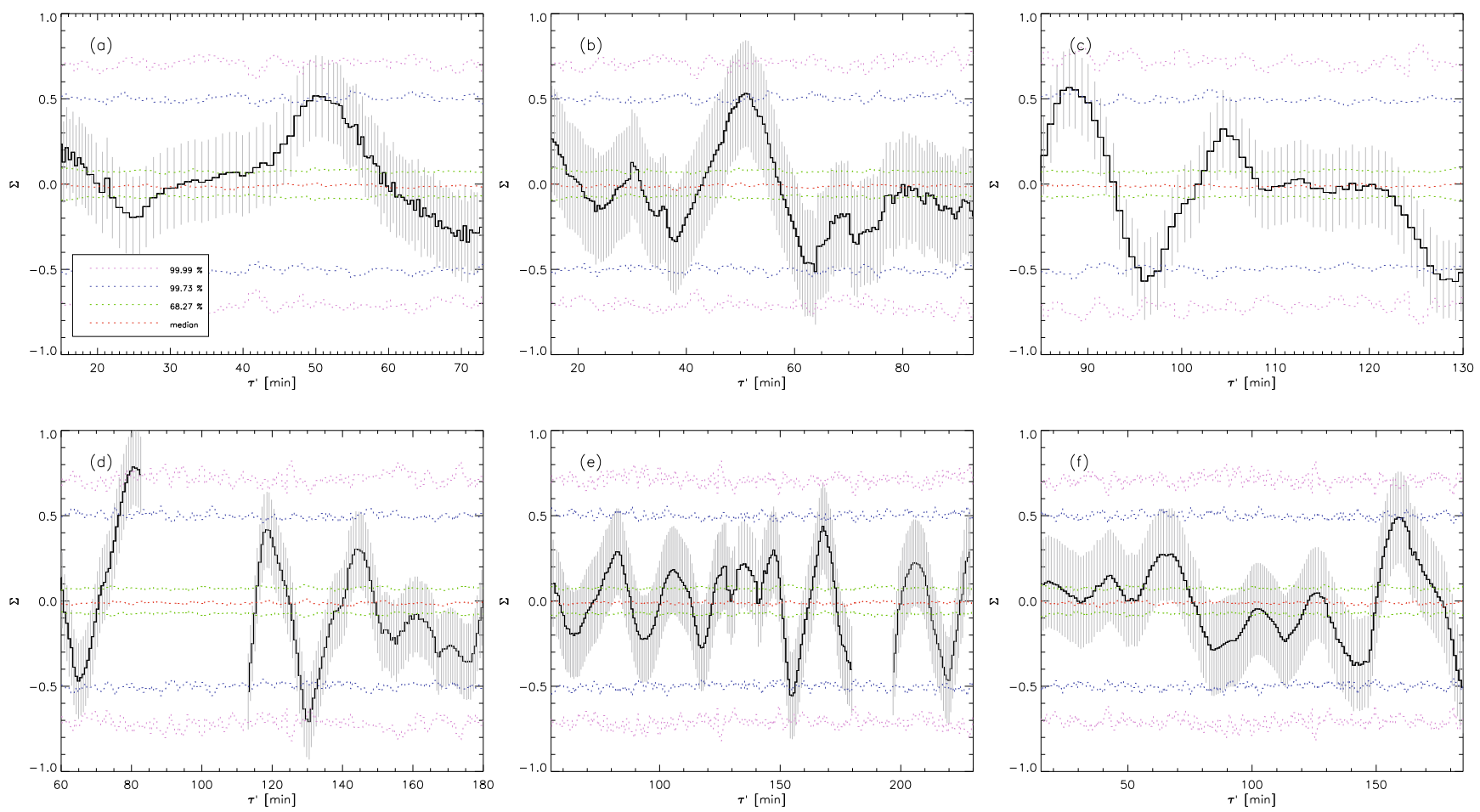

Fig. 21. Pattern recognition coefficients as a function of time for our sample of observations (13 June 2004 a); 30 July 2005 b); 1 June 2006 c); 15 May 2007 d); 17 May 2007 e) and 28 May 2008 f)). Dotted lines show the median (red), 68.3\% (green), 99.8\% (blue) and 99.99\% (violet) significance levels derived from $10^{4}$ random red noise light curves.

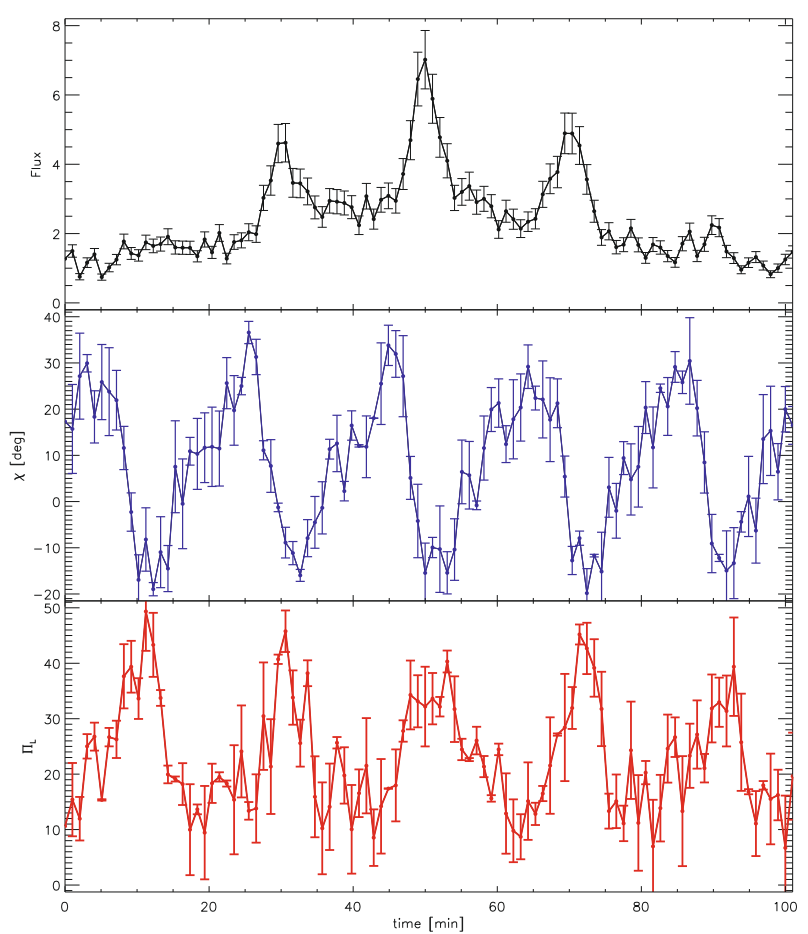

Fig. 22. A selected 100 min window of the white noise added simulated light curves of an orbiting spot plus a temporary variability in the accretion disk. The flux density is presented in arbitrary units (top). Middle and bottom panels show the changes in the angle (degree) of polarization. The spin of the black hole is set to be $a=0.5$ and the inclination fixed on $i=60^{\circ}$.

amount of change in the polarization angle depends on the compactness of the source, its position relative to the black hole, the
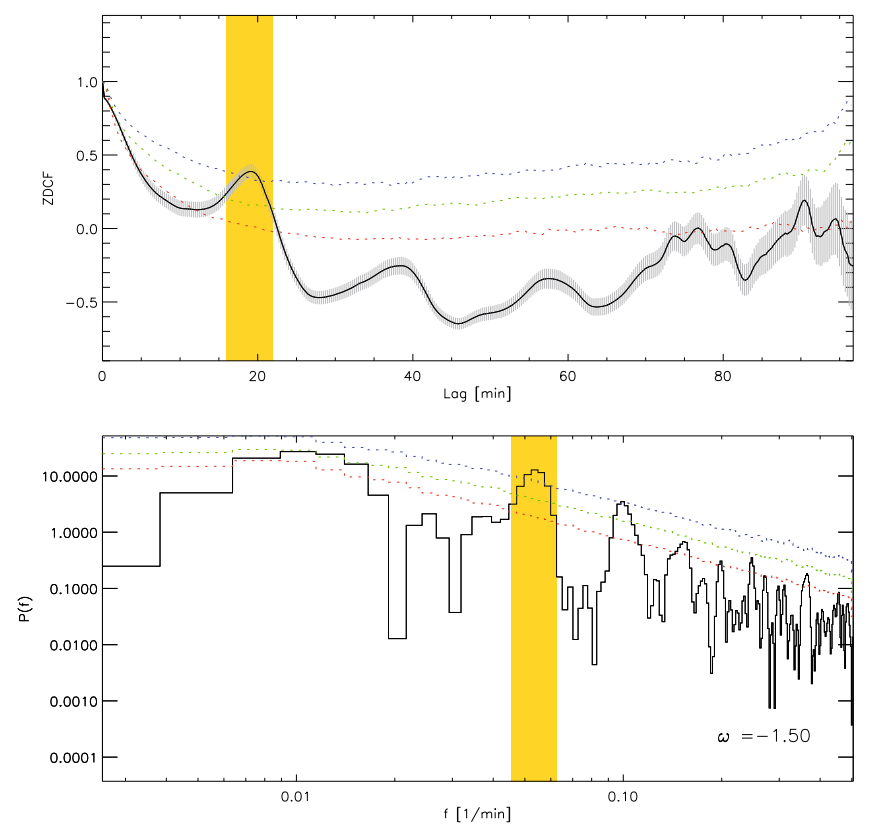

Fig. 23. Same as Figs. 3 and 5 for the simulated light curves of Fig. 22. The top shows the autocorrelation of the flux while the bottom shows the Lomb-Scargle periodogram. The colored regions indicate the position of the peaks corresponding to the $0.8-1.2 \times r_{\text {mso }}$ orbital time scales of a Kerr black hole with spin 0.5. Dotted lines show the median (red), $68.3 \%$ (green) and $99.8 \%$ (blue) confidence levels of the red noise.

inclination of the observer and the spin of the black hole. On the other hand, if the flare-emitting region is a deformed pattern extending in both radial and azimuthal directions, the swing in the polarization angle will not be strong. It is because of the fact 


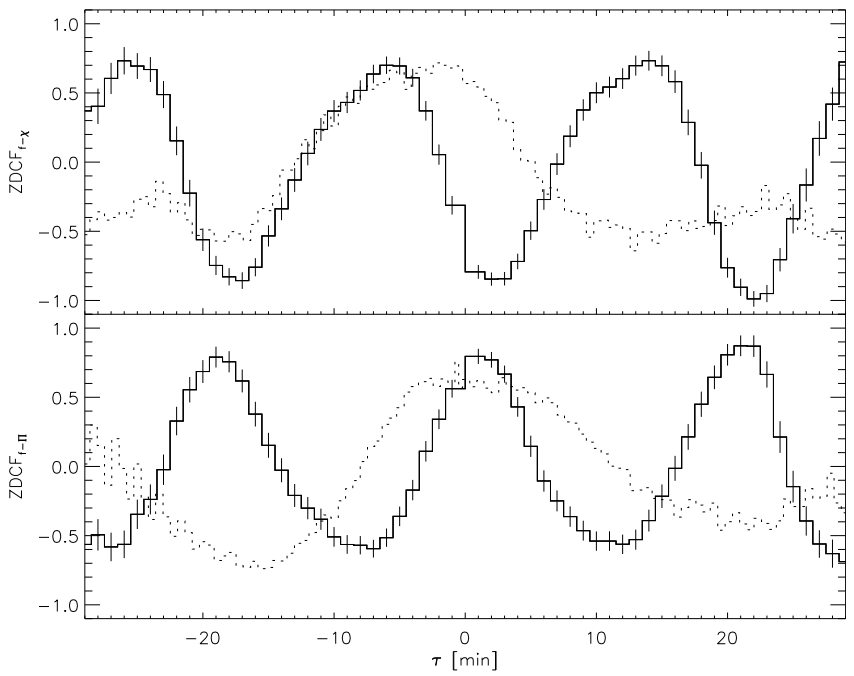

Fig. 24. Top (bottom): Cross correlation between the total flux and polarization angle (degree) of the simulated light curve (Fig. 22). Dotted lines indicate the corresponding cross correlation functions for the 30 july 2005 flare as the case most similar to the predictions of the hot spot model.

that in each point of the light curve the polarization angle is the average of photons that come from different parts of the disk and have a different polarization angle (Fig. 31). As a result, the swings in the angles cancel each other out, and no significant swing will be observed.

The presence of changes in the degree of the NIR polarization and the swings in the observed polarization angle simultaneous to the flux magnifications supports the idea that the geometrical shape of the sources is dominated by compact azimuthal asymmetries rather than by radially extended spiral patterns (see also discussion in Sect. 6 about the differences in the centroid motions of these two types of geometries). Here we must note that as Tagger et al. (2006) have mentioned, a second initial configuration was used in their simulations (simulation no. 2, Tagger et al. 2006). In that set-up, a clump of matter starts spiraling towards the black hole and produces the spiral Rossby wave. There, the ratio of the surface brightness between the hot core moving radially inward and the tail which is produced plays a critical role in the resulting light curves. If the hot core is dramatically brighter than the tail, there is no practical difference between this scenario and the orbiting spot model. For a complete polarization study of Rossby wave instabilities, one needs simulated profiles of MHD surface densities as a function of time, which is beyond the scope of this paper.

\section{Magnetic field structure and geometrical orientation of the system}

In our simulations the structure of the magnetic field lines according to the black hole/accretion disk system can be controlled by a set of parameters $[\eta, \psi]$. Of special interest are two extreme configurations in which the envelope of the accelerated electrons is located inside the accretion disk with a global toroidal magnetic field (model I: $\left[\eta=\frac{\pi}{2}, \psi=\frac{\pi}{2}\right]$ ) or inside a region with magnetic field lines perpendicular to the disk (model II: $[\eta=0, \psi=0])$. The latter can be interpreted as the spot being located at the tip of a jet with magnetic field lines aligned parallel to its symmetry axis (see Fig. 32).

We assumed that the magnetic field inside the accretion disk is dominated by the toroidal component. This structure is compatible with the results of several MHD simulations (Hawley \& Balbus 1991; Hirose et al. 2004; De Villiers et al. 2003). However, in our case it is not clear if the cloud of accelerated electrons is located inside the accretion disk, somewhere above it in the corona, or even inside the tip of a possible short jet/wind. In fact, there is a possibility that a source structure in which an accretion disk is associated with a short jet/collimated outflow can explain the multi-wavelength behavior of Sgr A* (Markoff et al. 2001; Yuan et al. 2002; Eckart et al. 2005, 2006a, 2008ac). Here we discuss how different orientations of the magnetic field lines inside the emitting region affect the resultant physical parameters that one can extract via fitting the model to the observed data.

The KY code allows us to simulate light curves for a wide range of free parameters, so one can fit the model parameters to the actual observed data (Meyer et al. 2006a,b, 2007; Eckart et al. 2006a,b, 2008). For this purpose, simulations have been carried out, as they cover a wide enough range of possible inclinations $\left(0.1^{\circ} \leq i \leq 85^{\circ}\right)$, the initial degree of linear polarization $\left(0 \% \leq \Pi_{\mathrm{L}} \leq 70 \%\right)$, possible combinations of $\eta$ and $\psi$, and different orientations of the whole system on the sky $\left(0^{\circ} \leq \Theta \leq 90^{\circ}\right)$. Here $\Theta$ defines the direction of the normal to the accretion disk projected on the sky. The spot has an initial radius of $1 R_{\mathrm{s}}$ and orbits very closely to the marginally stable orbit of a Kerr black hole $(a=0.5)$. The dimensionless shearing time scale is fixed to be $2.0\left(\tau_{\mathrm{sh}}=2.0\right)$, and the ratio of the surface brightness of the spot to the torus is set to five. We have chosen these values according to existing results of several fits to the NIR flares (Meyer et al. 2006a,b; Eckart et al. 2006b, 2008).

As a first step, we focus on model I ([ $\left.\left.\eta=\frac{\pi}{2}, \psi=\frac{\pi}{2}\right]\right)$. In order to find the fit with best $\chi_{\text {red }}^{2}$ we have carried out a grid search in a $\left(i-\Theta-\Pi_{\mathrm{L}}\right)$ parameter space. The steps for $i, \Theta$ and $\Pi_{\mathrm{L}}$ have been chosen to be $5^{\circ}, 5^{\circ}$ and $5 \%$, respectively. The least $\chi_{\text {red }}^{2}$ value was achieved for a high inclination angle $\left(i=60^{\circ}\right)$, $\Theta=15^{\circ}$ and highly polarized source $\left(\Pi_{\mathrm{L}}=50 \%\right)$ (see Fig. 33 and Table 2). The grid search has been performed on $\Pi_{\mathrm{L}}, i$ and $\Theta$ for the models I and II. The free parameters in model III are $\Pi_{\mathrm{L}}, i, \eta$ and $\psi$. Figure 34 shows the resultant possible range for the orientation of the correlated outflow/wind in this scenario. Interestingly, the observed position of the mini-cavity lies well within this interval. Mužić et al. (2007) have shown how a collimated outflow from the position of $\mathrm{Sgr} \mathrm{A}^{*}$ can describe the observed motion of filamentary structures and the mini-cavity, according to their observations in the NIR $L^{\prime}$-band. This observational evidence supports the idea that the NIR flares actually originate in accelerated electrons within an accretion disk.

For model II $([\eta=0, \psi=0])$, the envelope of relativistic electrons has been located somewhere in the bottom of an outflow. The least $\chi_{\text {red }}^{2}$ achieved for this model and related parameters are presented in Table 2. Possible directions of this jet structure are depicted in Fig. 34. As one can see, $\chi_{\text {red }}^{2}$ values and observational facts support the idea that the NIR photons originate in the inner parts of an accretion disk rather than in an outflow (Thorne 1994; Blandford 2001).

One can also invert the question and try to constrain the magnetic field structure of the emitting region by fixing the direction of the outflow in the plane of the sky (model III). For this purpose, $a \chi_{\text {red }}^{2}$ search has been carried out, assuming that the normal to the disk is fixed at $\Theta=30^{\circ}$. This is the best value that can fit the observed position of the mini-cavity (Mužić et al. 2007). By 


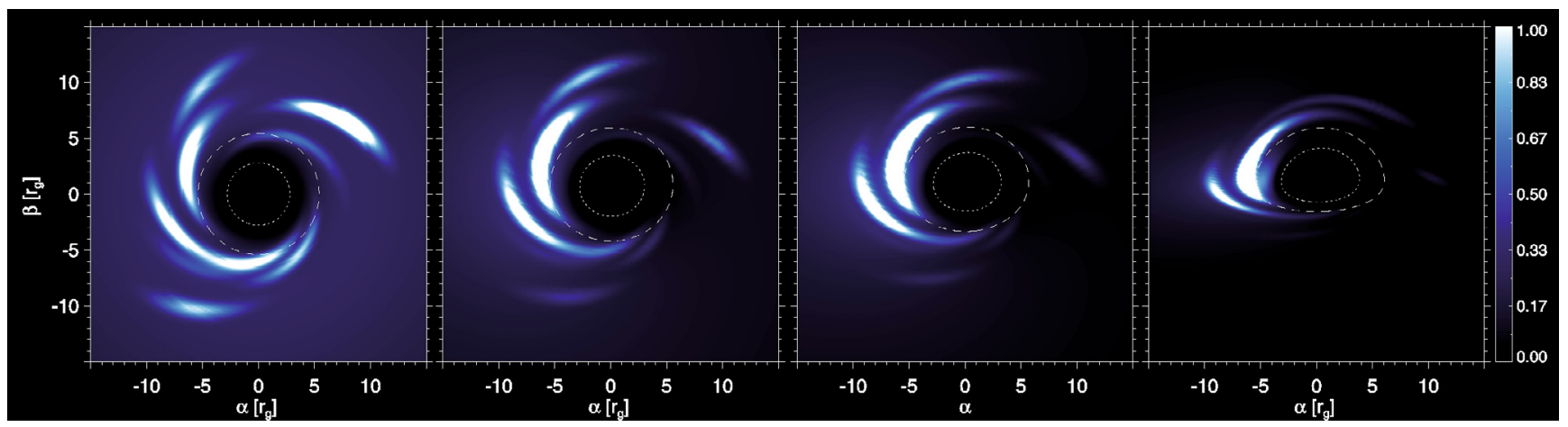

Fig. 25. Same as Fig. 16 for the multi-component scenario. The observer's inclination is set to $0^{\circ} \mathbf{a}$ ), $30^{\circ} \mathbf{b}$ ), $45^{\circ} \mathbf{c}$ ) and $70^{\circ}$ d) (see the discussion in Sect. 3.1 and Table A.2 in Eckart et al. 2008a).

Table 2. Final parameters resulting from the best $\chi_{\text {red }}^{2}$ fit to the NIR flare observed on 30 july 2005 .

\begin{tabular}{lcccccccccc}
\hline \hline Model & $a$ & $\begin{array}{c}\eta \\
{[\mathrm{deg}]}\end{array}$ & $\begin{array}{c}\psi \\
{[\mathrm{deg}]}\end{array}$ & $\begin{array}{c}r_{0} \\
{\left[r_{\mathrm{g}}\right]}\end{array}$ & $\begin{array}{c}r_{\text {spot }} \\
{\left[r_{\mathrm{mso}}\right]}\end{array}$ & $R$ & $\begin{array}{c}\Pi_{\mathrm{L}} \\
{[\%]}\end{array}$ & $\begin{array}{c}i \\
{[\mathrm{deg}]}\end{array}$ & $\begin{array}{c}\Theta \\
{[\mathrm{deg}]}\end{array}$ & $\chi_{\text {red }}^{2} \pm 1$ \\
\hline I & 0.5 & 90 & 90 & 4 & 1.1 & 5 & $50 \pm 10$ & $60 \pm 15$ & $15 \pm 20$ & $1.63 \pm 1$ \\
II & 0.5 & 0 & 0 & 4 & 1.1 & 5 & $50 \pm 10$ & $65 \pm 10$ & $110 \pm 15$ & $2.95 \pm 1$ \\
III & 0.5 & $78.56 \pm 12$ & $72 \pm 18$ & 4 & 1.1 & 5 & $60 \pm 10$ & $50 \pm 20$ & 30 & $1.54 \pm 1$ \\
\hline
\end{tabular}

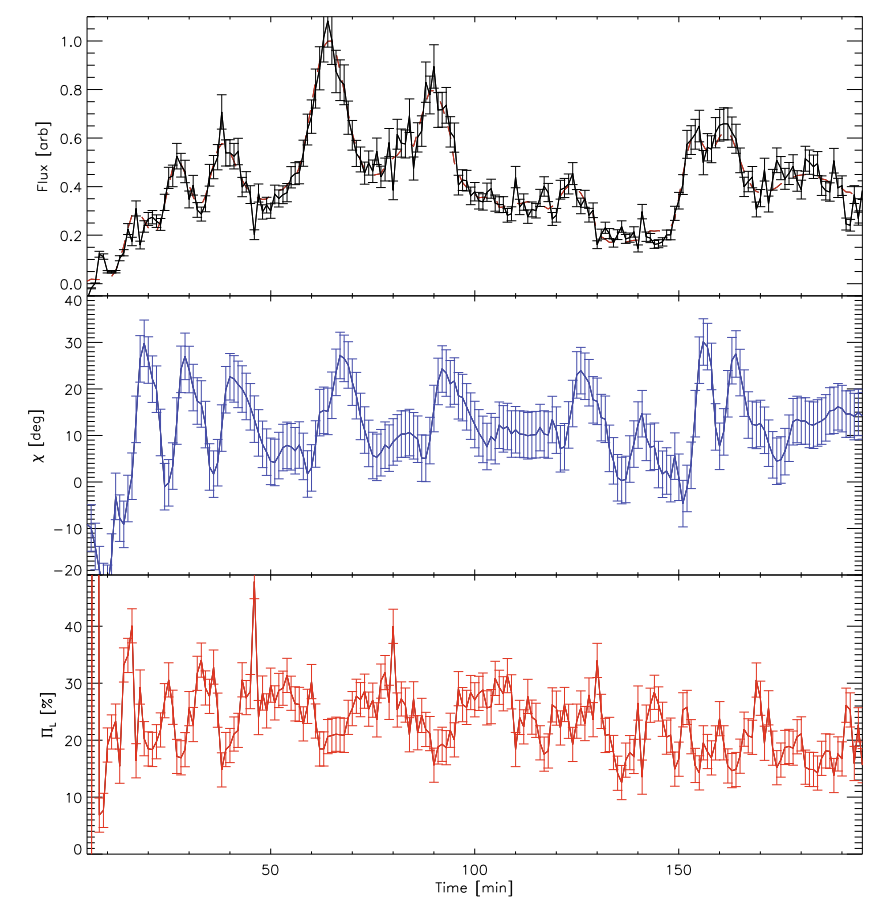

Fig. 26. Same as Fig. 22 but a selected 200 min-window of the multi component scenario.

a good approximation, the best fit is achieved for a toroidal magnetic field structure (see Table 2). This shows that if any future observations reveal traces of an outflow from Sgr A*, NIR polarimetry could be used as a tool to constrain its magnetic field structure.
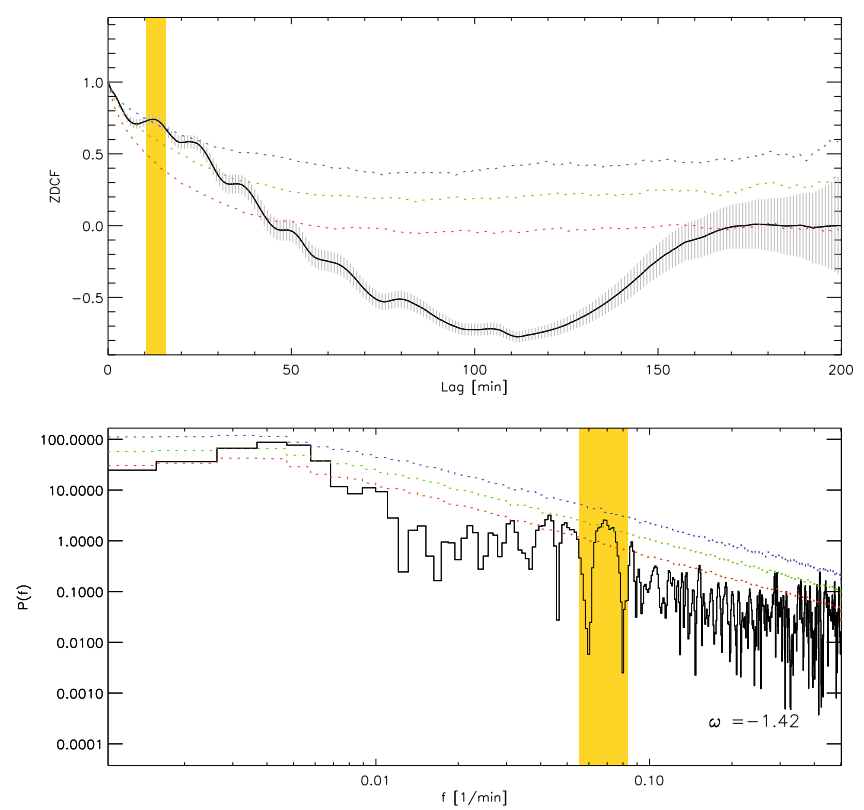

Fig. 27. Same as Figs. 23 for the multi-component scenario (Fig. 26).

\section{Next generation of NIR interferometry}

According to its proximity, Sgr $\mathrm{A}^{*}$ is known as the best candidate for studying the details of physical processes on the event horizon scales (Falcke et al. 2000; Bromley et al. 2001). VLT and Keck telescopes have already achieved angular resolutions as high as 40 mas in their imaging mode (Genzel et al. 2003; Eckart et al. 2004, 2006a,b; Ghez et al. 2005). In interferometric mode these telescopes can observe with resolutions of the order of only 


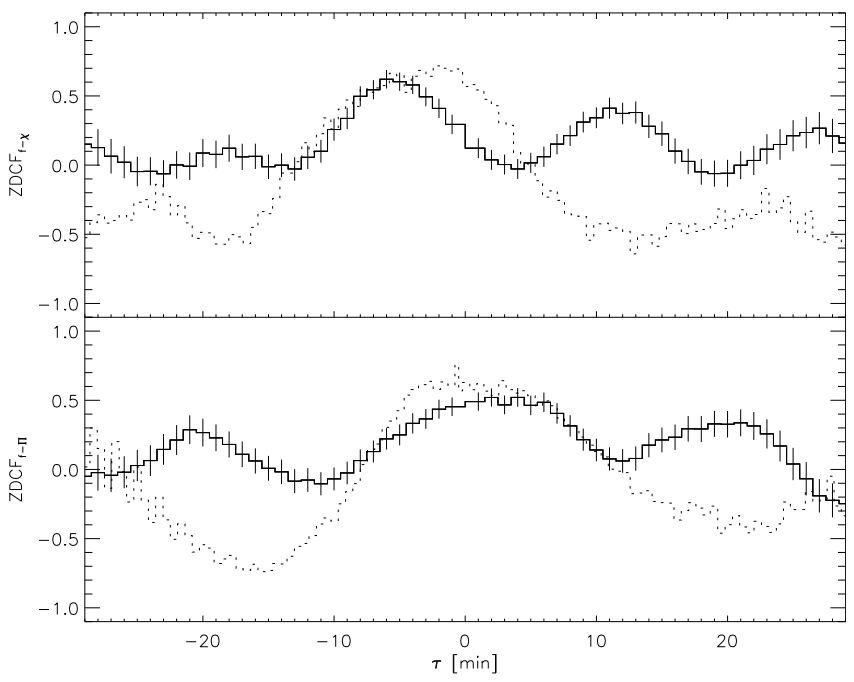

Fig. 28. Same as Figs. 24 for the multi-component scenario (Fig. 26).

a few mas. The next generation of VLT Interferometer instrument, namely GRAVITY, would be able to achieve resolutions of the order of $10 \mu$ as in its phase-reference tracking mode. GRAVITY will try to measure the position of the lumicenter of the flares with respect to a reference source. For such a measurement the $1 \sigma$ uncertainty is $\pm 10 \mu$ as with an integration time of five minutes on an assumed $K=15$ pointsource. The real necessary integration time for that precision is of course directly dependent on the magnitude of the flare. This resolution is high enough to track the centroid motion of NIR images of Sgr A* with a resolution of the order of one Schwarzschild radius (Eisenhauer et al. 2005, 2008; Gillessen et al. 2006). These unique features make the next generation of VLTI a perfect instrument for high accuracy astrometry of the matter around Sgr A*. Recently, Doeleman et al. (2008) proposed a VLBI configuration with a high enough time resolution to possibly track the centroid motion of sub-mm images of Sgr A* with even higher angular resolution. In this section we present the results of our study on centroid motion of the NIR image of Sgr A*.

Some predictions of the centroid motions related to the hot spot model have already been studied by several authors (Broderick \& Loeb 2006a,b; Paumard et al. 2006; Zamaninasab et al. 2008b; Hamaus et al. 2009). In previous works it has been mainly assumed that the spots preserve their shape in time. This assumption has been based mostly on the presence of strong magnetic field lines or "unknown mechanisms" (Hamaus et al. 2009). We will show here that it is actually very crucial that the effects of gravitational shearing inside the accretion disk are taken into account, since the predicted results can change dramatically. The other very important fact to be considered in the simulations is due to the NIR photons which come from the body of the accretion disk and confuse with the spot's emission. Since GRAVITY can only achieve its $10 \mu$ as resolution in the phasereference tracking mode and not in the imaging mode, even a constant confusion from the (thermal) electrons inside the accretion disk can not be reduced.

Figure 35 represents how these two parameters mentioned can affect the expected centroid tracks and may present a complication in detecting the plasma structure close to the event horizon of Sgr A*. The simulated paths in Fig. 35 belong to a configuration where the observer is aligned approximately face-on $\left(i \simeq 0^{\circ}\right)$. This is the inclination in which the clearest wobbling of the centroid is expected to be seen. Figure $35 \mathrm{a}$ shows that the position wanders of the center of the images are large enough to be detected easily if GRAVITY could achieve its five minutes time-resolution (simulated points). Panels b and c in Fig. 35 show how gravitational shearing changes the centroid track. One can see in particular that in the case of pure Keplerian shearing the change in the center is detectable for approximately only six points. The second row (Fig. 35, panels d)-f)) shows the result of the simulated tracks including the effect of confusion due to the NIR photons coming from the inner parts of the accretion disk.

Figure 36 shows the centroid paths of spots orbiting in a mild shearing environment $\left(\tau_{\mathrm{sh}}=2.0\right)$ for three different inclinations $\left(i=0.1^{\circ}, 30^{\circ}, 60^{\circ}\right)$ in presence of confusion from the torus. Panel $c$ shows that the detection of any position wander becomes even more difficult in the case of a shearing spot observed at high inclinations. The bottom row in Fig. 36 shows the centroid motion resultant from simulations including several emitting components (Fig. 25). The photons originated from the spot are highly polarized, while the radiation from the torus is weakly polarized. This fact may lead us to a solution for compensating for this kind of confusion. Figure 37 shows how different the apparent images of the flares are when they are observed in total flux or two orthogonal polarized channels. One sees that the different centroid paths can be revealed if GRAVITY could achieve its $10 \mu$ as resolution in the polarimetry mode Fig. 38 .

To summarize the results of this section, one can say that a non-detection of a position wander in NIR images of Sgr A* will not rule out a spotted disk scenario. On the other hand, a clear detection of such a position wander by GRAVITY would lead us to strongly favor the spot model and would open a new era in studying the physics of high gravitational regimes.

\section{Summary and conclusion}

We used a sample of NIR flares of Sgr A* observed in polarimetry mode to study the nature of the observed variability. Using the z-transformed discrete correlation function algorithm, we found a significant correlation between changes in the measured polarimetric data and total flux densities. This provides evidence that the variations probably originate from inner parts of an accretion disk while a strong gravity's effects are manifested inside them.

In order to obtain this information polarization data is indispensable since the corresponding signals are very difficult or even impossible to extract from simple total intensity light curves only (Do et al. 2008). The presence of significant signals from orbiting matter then calls for detailed modeling of the NIR polarized light curves in order to analyze the distribution of the emitting material and the magnetic field structure within the accretion disk (e.g. Eckart et al. 2006a, 2008a; Meyer et al. 2006a,b, 2007).

In addition, the pattern recognition algorithm we employed in this paper is an efficient tool to search for flare events that carry the signature of strong gravity in light curves which are significantly longer than the orbiting time scale over which such an event can typically occur. Other methods that make simultaneous use of the entire light curve (like e.g. the Lomb-Scargle algorithm) tend to dilute these signals and lower the possibility for significant detections dramatically (Do et al. 2008; Meyer et al. 2009). 


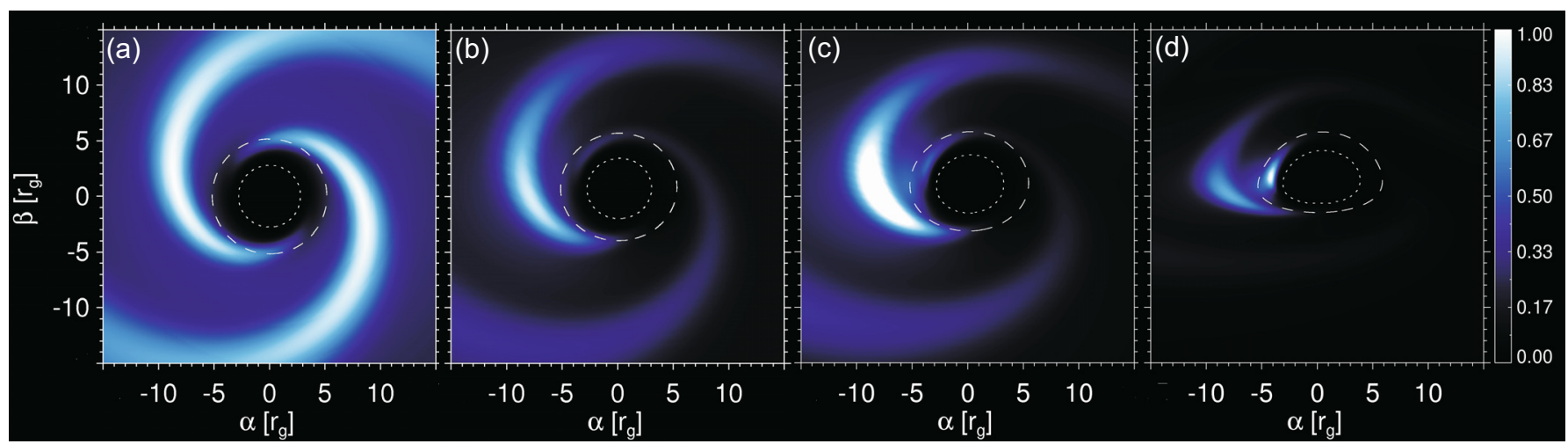

Fig. 29. Snapshots of the orbiting spiral pattern as they appear to a distant observer looking along a line of sight inclined by $0^{\circ}$ a), $\left.30^{\circ} \mathbf{b}\right), 45^{\circ}$ c) and $70^{\circ}$ d) (relative to the normal to the disk). See also the caption of Fig. 15.
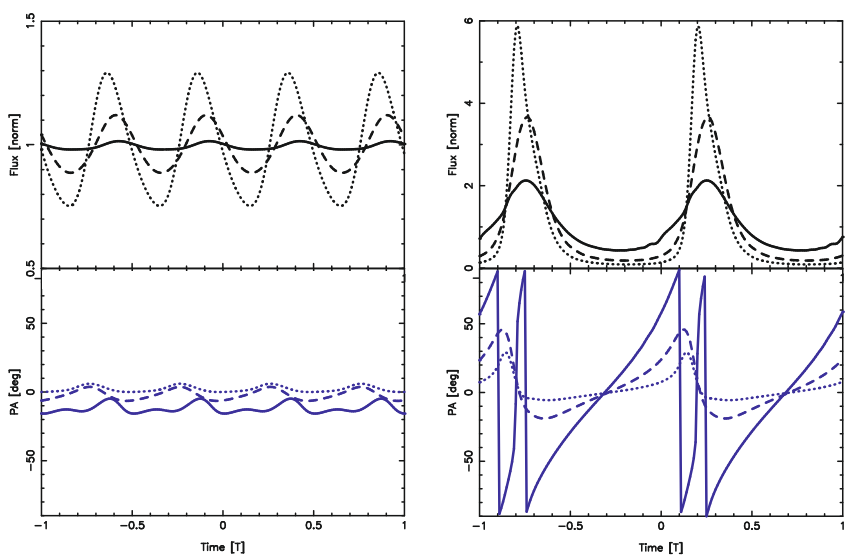

Fig. 30. Light curves of the normalized flux (top, black) and polarization angle (bottom, blue) observed at infinity, obtained form general relativistic modulations associated with a spiral pattern (left) or a compact spot $(r i g h t)$. Light curves are shown for three different inclinations: $i=30^{\circ}$ (solid line), $i=60^{\circ}$ (dashed line) and $i=80^{\circ}$ (dotted line). The modulations happen twice as often for the spiral pattern than for the spot in the same interval, according to the existence of two symmetrical arms. Flux values are normalized according to their average values. The spin of the black hole is set to be zero $(a=0)$.

In order to constrain the physical properties of the emitting region we employed a relativistic disk model with azimuthal over-densities of relativistic electrons. A combination of a synchrotron mechanism and relativistic amplifications allows us to fit the real observed data and make predictions about astrometric parameters of the accretion disk around Sgr A*. The modeled light curves show the same correlation between the flux and polarimetric data as the one deduced from observations.

Simulations have been carried out in a way that they cover a wide range of parameters, including the effects of gravitational shearing inside the accretion disk, the heating and cooling time scales, the inclination and the spin of the black hole. It is discussed how the observed swings in the polarization angle support the idea of a compact source for the emission, instead of radially extended spiral shapes. Furthermore, we present a model in which the observed NIR polarization angle can lead to confining the expected region for the expected outflow/wind from Sgr A*. The model also predicts that when observations will be
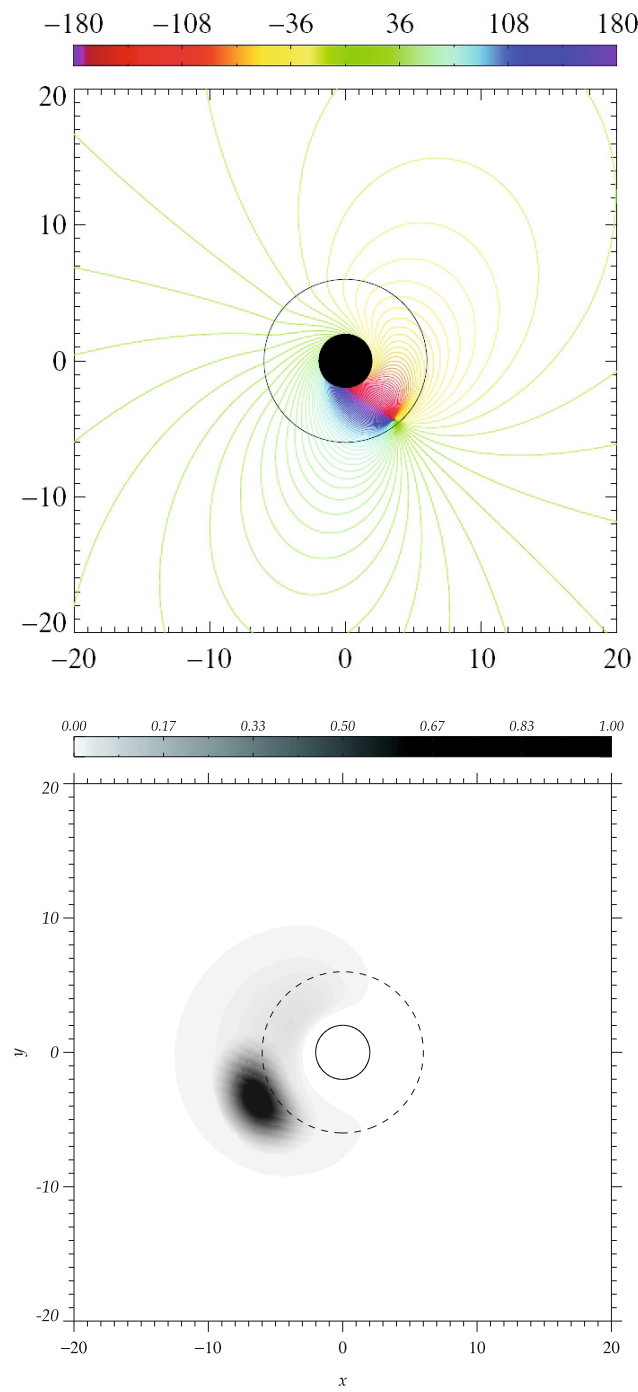

Fig. 31. Top: Contour graphs showing the changes in the polarization angle due to general relativistic effects. Bottom: Spatial emissivity distribution of a compact hot spot. The observer's inclination is $i=45^{\circ}$ and the black hole is assumed to be spin-less. The observer is located on the top of the pictures. The innermost stable orbit is shown in both images (solid line in top image and dashed line in the bottom). The graphs are represented in the coordinates $x=r \cos \phi, y=r \sin \phi$ in the equatorial plane where $r$ and $\phi$ are Boyer-Lindquist coordinates. Units are $r_{\mathrm{g}}$ in the $\mathrm{x}-\mathrm{y}$ coordinates. 
to the

observer

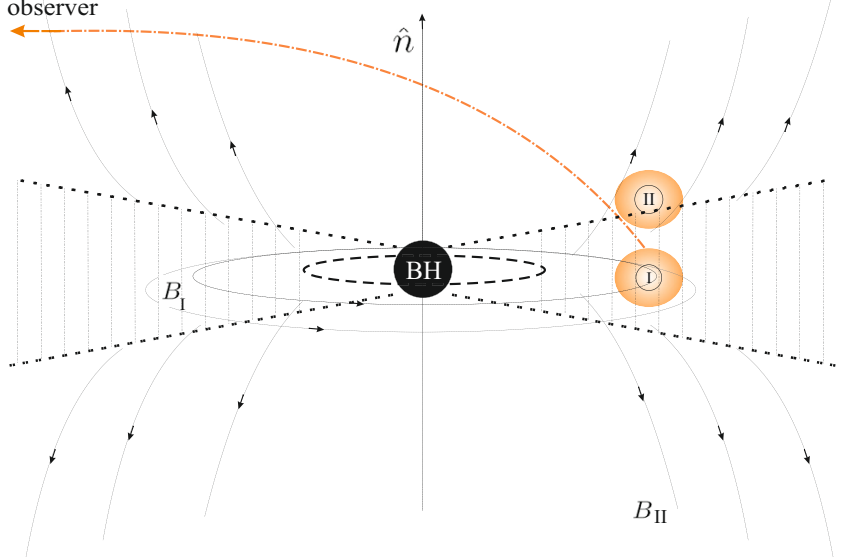

Fig. 32. A sketch of the model (not to scale) of two extreme cases of global magnetic field configuration. Model I: the localized flare happens inside the accretion disk, where the dominant component of the magnetic field is toroidal $\left(B_{\mathrm{I}}\right)$. Model II: over-density of the accelerated electrons happens at the tip of a possible short jet (wind). Magnetic field lines $\left(B_{\mathrm{II}}\right)$ are assumed to be elongated toward the axial symmetry axis of the system, parallel to the normal to the disk $(\hat{\boldsymbol{n}})$.

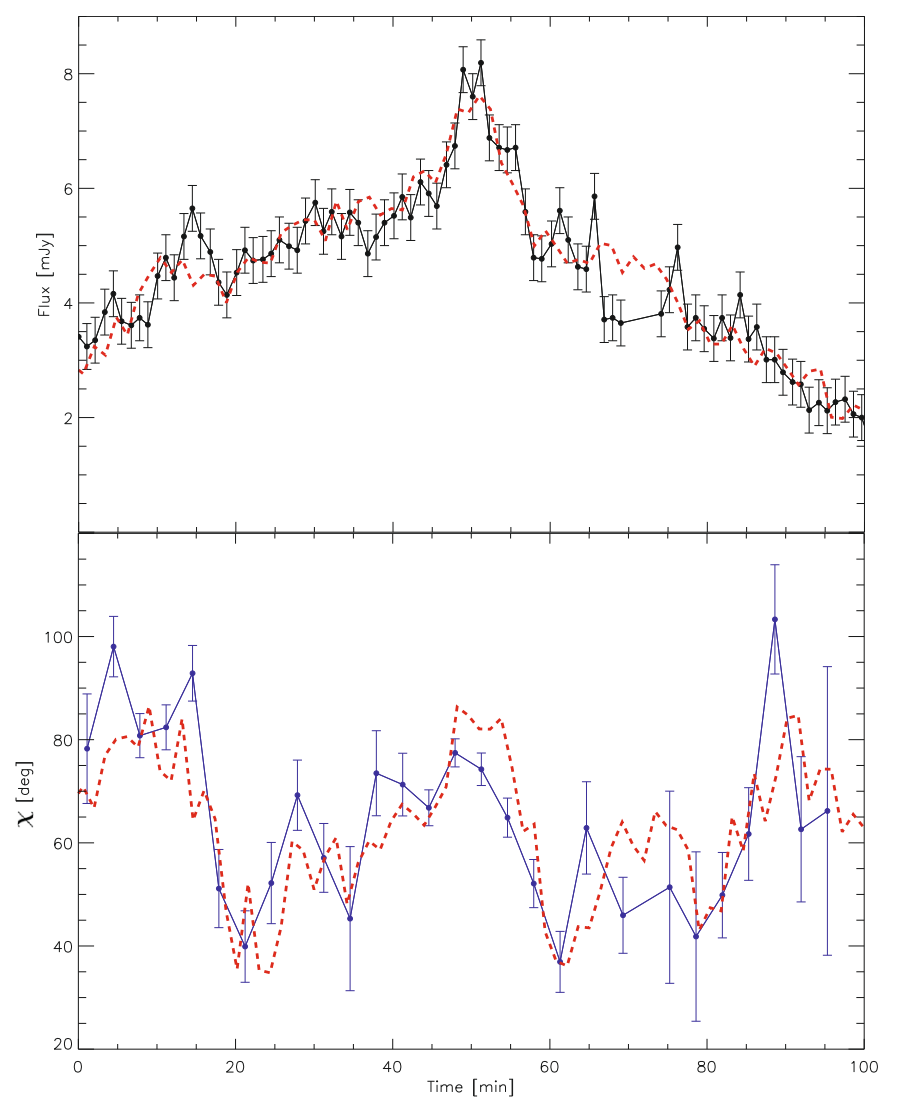

Fig. 33. Best fit achieved for the flux and polarization angle of the 30 july 2005 flare. The points and error bars represent the observation, red dashed lines show the model with Gaussian noise being added. The parameters are presented in Table 2.

able to resolve the position of such an outflow, the magnetic field structure inside the accretion disk could be confined.

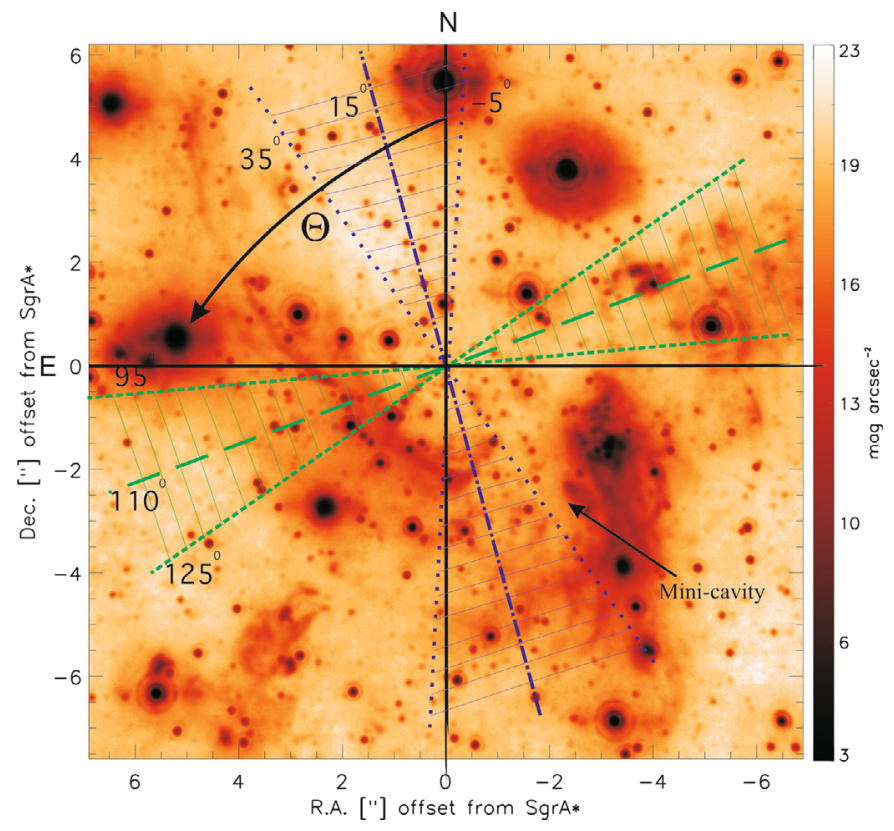

Fig. 34. The resulting orientations for the direction of possible collimated outflow from Sgr A* $(\Theta)$ for model I (blue dashed-dotted line) and model II (green long dashed line). The shaded regions indicate the range of possible values of $\Theta$ for $\chi_{\text {red }}^{2} \pm 1$ interval. The background image shows the Galactic Center environment in NIR $L^{\prime}$ band. Sgr A* is located at the center of the image. The position of the mini-cavity (indicated by an arrow) coincides well with the predictions of model I.

Finally, the centroid paths of the NIR images are discussed. In comparison with the results by Broderick \& Loeb (2006a,b) and Hamaus et al. (2009), we have shown that the geometrical structure of the emitting region (elongation of the spot according to the Keplerian shearing, multi-component structures, spiral arms, confusion caused by the radiation from the hot torus) can affect the expected centroid tracks. While all the mentioned geometries are able to fit the observed fluxes, we show how the future NIR interferometer GRAVITY on the VLT can break these degeneracies. The results of simulations propose that focusing GRAVITY observations on the polarimetry mode could reveal a clear centriod track of the $\operatorname{spot}(\mathrm{s})$. We conclude that even though a non-detection of centroid shifts can not rule out the multi-components model or spiral arms scenarios, a clear position wander in the center of NIR images during the flares will support the idea of bright long-lived spots orbiting occasionally around the central black hole. This possible detection opens a new window for testing the physics very close to the edge of infinity.

Acknowledgements. The authors would like to thank anonymous referee for helpful comments. Part of this work was supported by the German Deutsche Forschungsgemeinschaft, DFG via grant SFB 494. M. Zamaninasab, and D. Kunneriath are members of the International Max Planck Research School (IMPRS) for Astronomy and Astrophysics at the MPIfR and the Universities of Bonn and Cologne. N. Sabha acknowledges support from the Bonn-Cologne Graduate School of Physics and Astronomy (BCGS). V.K. and M.D. acknowledge the Czech Science Foundation (ref. 205/07/0052). RS acknowledges the Ramón y Cajal program of the Spanish Ministry of Science and Innovation. 
M. Zamaninasab et al.: NIR flares of Sgr A*

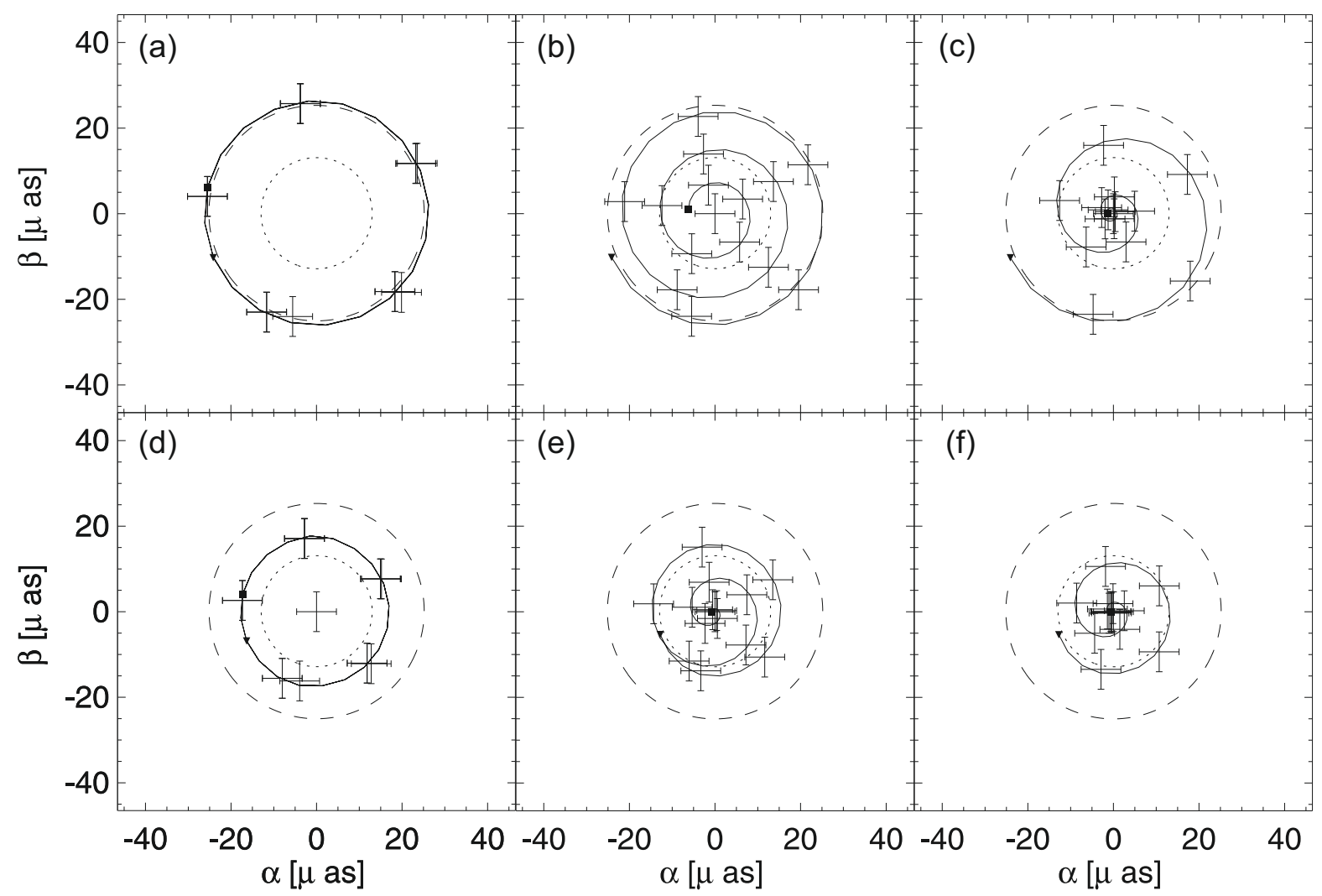

Fig. 35. The centroid motion of NIR images viewed from $0^{\circ}$ above the orbital plane for a Kerr black hole with a spin of 0.5 . The first row shows the paths for the background-subtracted images for different values of the gravitational shearing time scale. Each column shows $\tau_{\mathrm{sh}}$ values $\infty, 2.0$ and 1.0 from right to left. The second row shows the same paths for the images without the background subtraction. The error bars show the simulated positions as they are expected to be observed by GRAVITY ( $F W H M \sim 8.5 \mu$ as) with a time resolution of five minutes. The whole paths belong to three orbital times. A triangle indicates the beginning and a square the end of the track. Both coordinates are labeled in $\mu$ as units. The dotted and dashed lines indicate the position of the event horizon and the marginally stable orbit respectively.

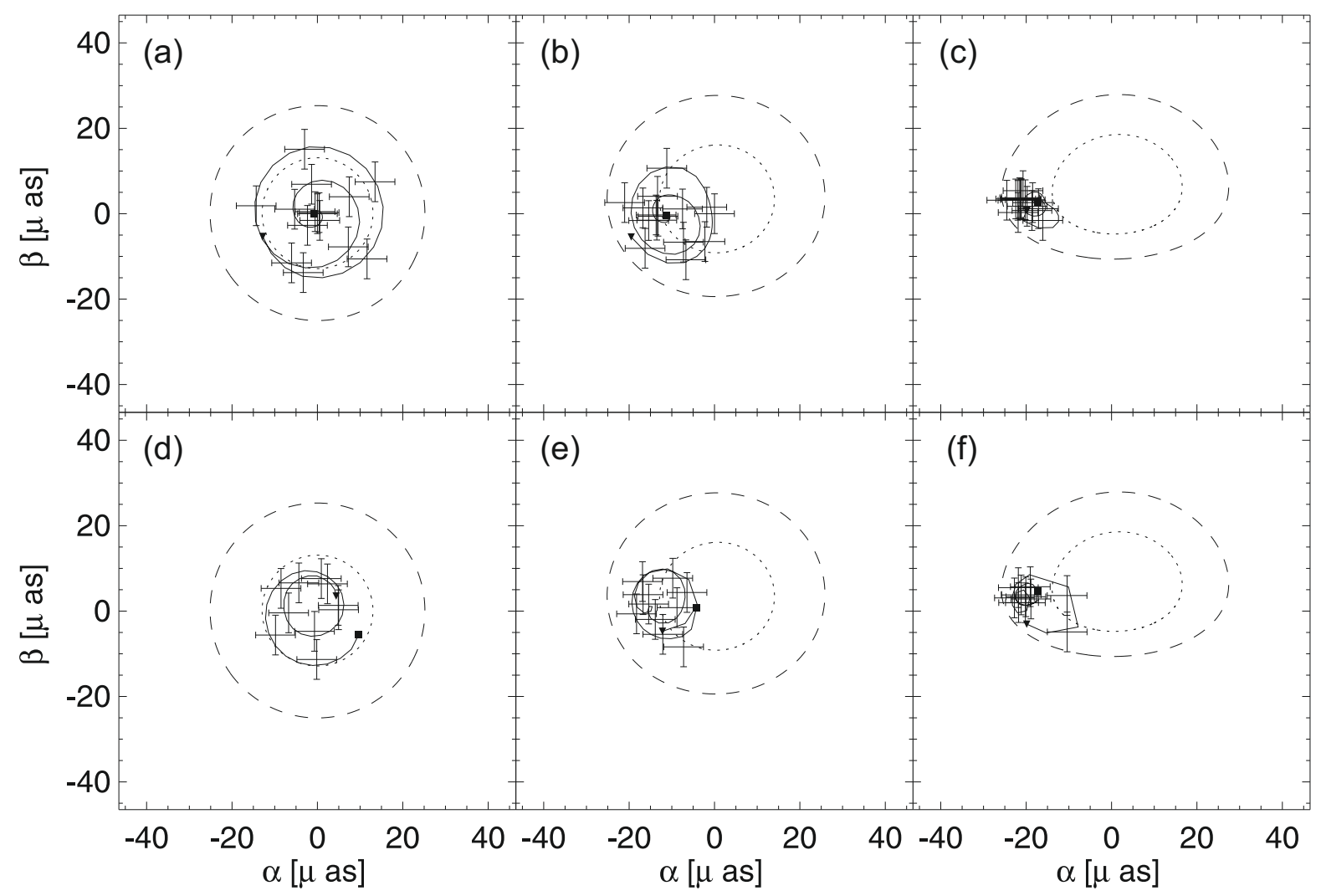

Fig. 36. Similar to Fig. 35 where different columns represent different values of inclination (from left to right: $\left.i=0.1^{\circ}, i=30^{\circ}, i=60^{\circ}\right)$. (a) $-\mathbf{c}$ )) shows the centroid track of an orbiting spot with $\tau_{\mathrm{sh}}=2.0$. (d) $\left.-\mathbf{f}\right)$ ) shows the centroid for a multi-component model (see Fig. 25). 


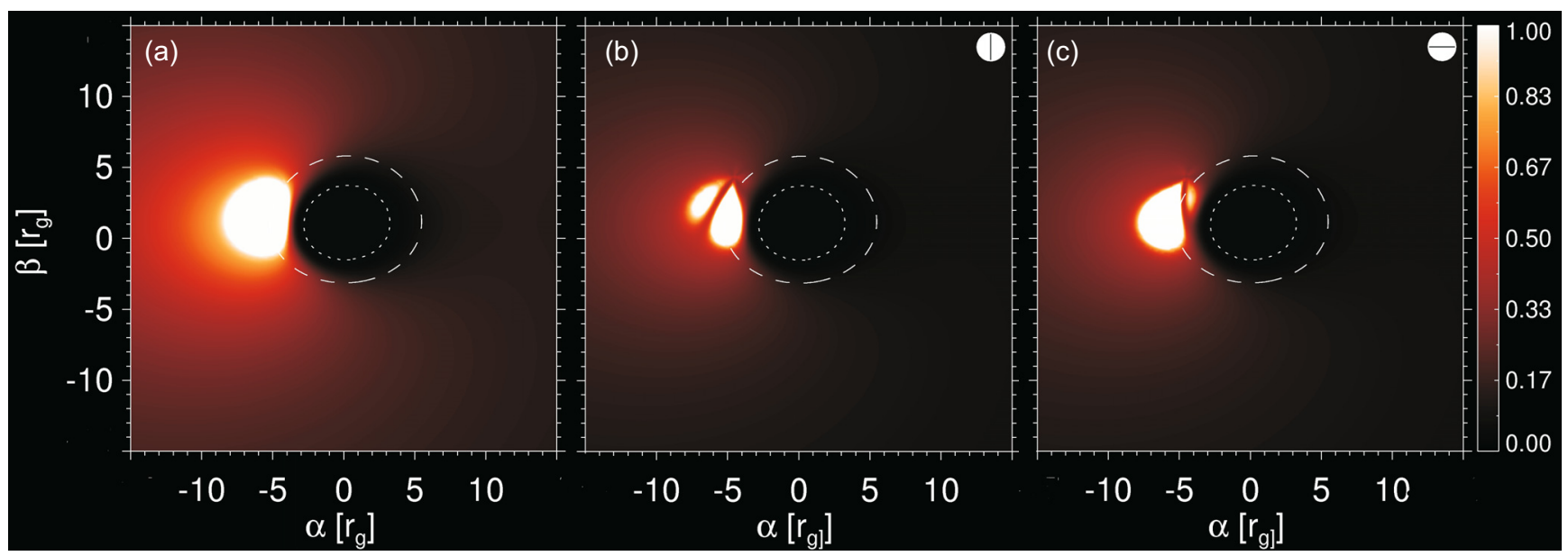

Fig. 37. Apparent images of a flare event for a distant observer looking along a line of sight inclined by $45^{\circ}$ in total flux a), ordinary b) and extra-ordinary polarized channels c).

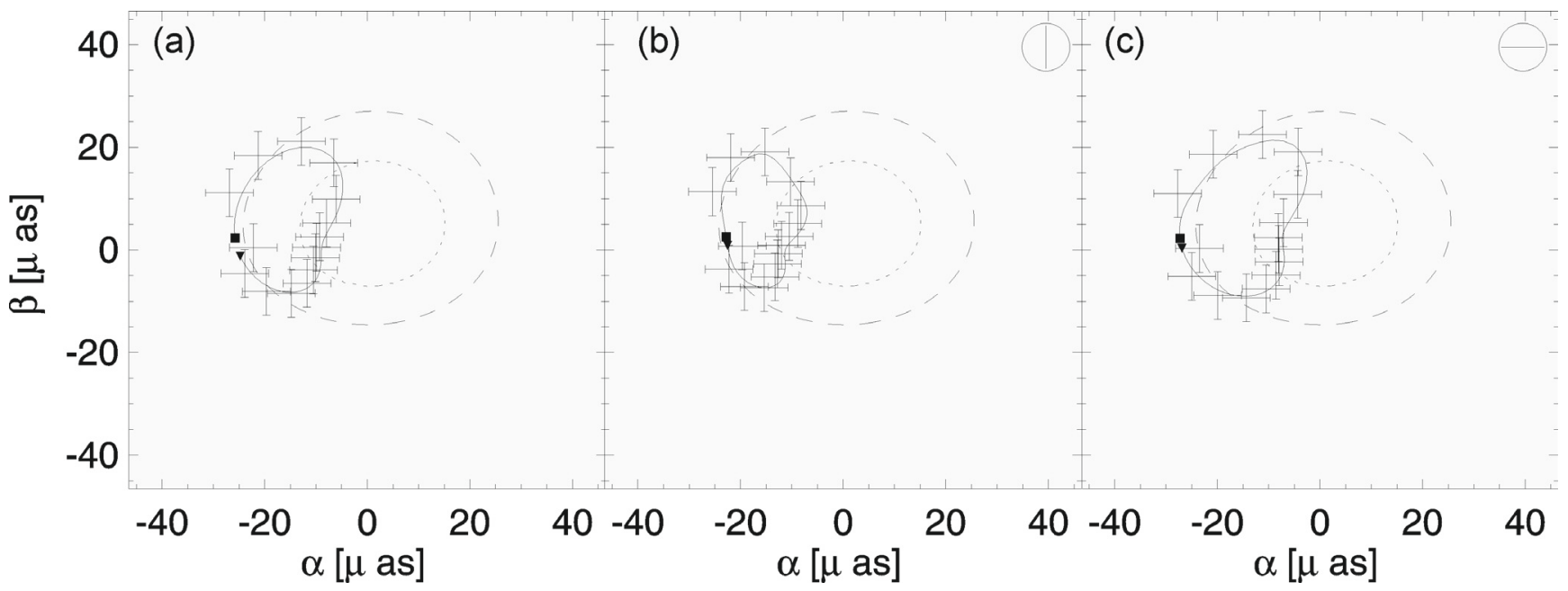

Fig. 38. Centroid paths for the same inclinations as Fig. 37 in the total flux a), ordinary b) and extra-ordinary c) polarized channels. See also caption of Fig. 35 .

\section{References}

Abramowicz, M. A., Bao, G., Lanza, A., \& Zhang, X.-H. 1991, A\&A, 245, 454 Abramowicz, M. A., Lanza, A., Spiegel, E. A., \& Szuszkiewicz, E. 1992, Nature, 356,41

Adams, F. C., \& Watkins, R. 1995, ApJ, v.451, 314

Alexander, T. 1997, ASSL, 218, 163

Armitage, P. J., \& Reynolds, C. S. 2003, MNRAS, 341, 1041

Baganoff, F. K., Bautz, M. W., Brandt, W. N., et al. 2001, Nature, 413, 45

Bardeen, J. M., Press, W. H., \& Teukolsky, S. A. 1972, ApJ, 178, 347

Belanger, G., Terrier, R., de Jager, O. C., Goldwurm, A., \& Melia, F. 2006, JPhCS, 54, 420

Blandford, R. D. 2001, Progress of Theoretical Physics Supplement, 143, 182

Broderick, A. E., \& Loeb, A. 2006a, ApJ, 636L, 109

Broderick, A. E., \& Loeb, A. 2006b, MNRAS, 367, 905

Bromley, B. C., Melia, F., \& Liu, S. 2001, ApJ, 555L, 83

Bursa, M., 2007, IAUS, 238, 333

Čadež, A., Calvani, M., Gomboc, A., \& Kostić, U. 2006, AIPC, 861, 566

Chan, C. 2009, ApJ, 704, 68

Chan, C., Liu, S., Fryer, C. L., et al. 2006, ApJ, 701, 521

Cunningham, J. M., \& Bardeen, C. T. 1973, ApJ, 183, 237

Devillard, N. 1999, ASPC, 172, 333

De Villiers, J., \& Hawley, J. F. 2003, ApJ, 589, 458

Diolaiti, E., Bendinelli, O., Bonaccini, D., et al. 2000, ASPC, 216, 623

Do, T., Ghez, A. M., Morris, M. R., et al. 2009, ApJ, 691, 1021

Doeleman, S. S., Fish, V. L., Broderick, A. E., Loeb, A., \& Rogers, A. E. E. 2008, ApJ, 695, 59

Dovčiak, M., Karas, V., \& Yaqoob, T. 2004, ApJS, 153, 205

Dovčiak, M., Karas, V., Matt, G., \& Goosmann, R. W. 2008a, MNRAS, 384, 361
Dovčiak, M., Muleri, F., Goosmann, R. W., Karas, V., \& Matt, G. 2008b, MNRAS, 391, 32

Eckart, A., \& Genzel, R. 1996, Nature, 383, 415

Eckart, A., \& Genzel, R. 1997, MNRAS, 284, 576

Eckart, A., Genzel, R., Ott, T., \& Schoedel, R. 2002, MNRAS, 331, 917

Eckart, A., Baganoff, F. K., Morris, M., et al. 2004, A\&A, 427, 1

Eckart, A., Baganoff, F. K., Schödel, R., et al. 2006a, A\&A, 450, 535

Eckart, A., Schödel, R., Meyer, L., et al. 2006b, A\&A, 455, 1

Eckart, A., Schödel, R., Meyer, L., et al. 2006c, ESO Messenger 125, 2

Eckart, A., Baganoff, F. K., Zamaninasab, M., et al. 2008a, A\&A, 479, 625

Eckart, A., Schödel, R., Baganoff, F. K., et al. 2008b, JPhCS, 131a, 2002

Eckart, A., Schödel, R., García-Marín, M., et al. 2008c, A\&A, 492, 337

Eckart, A., Baganoff, F. K., Morris, M. R. M., et al. 2009, A\&A, 500, 935

Eisenhauer, F., Schödel, R., Genzel, R., et al. 2003, ApJ, 597, L121

Eisenhauer, F., Genzel, R., \& Alexander, T., 2005a, ApJ, 628, 246

Eisenhauer, F., Perrin, G., Rabien, S., et al. 2005b, AN, 326, 561

Eisenhauer, F., Perrin, G., Brandner, W., et al. 2008, SPIE 7013E, 69

Falanga, M., Melia, F., Tagger, M., Goldwurm, A., \& Bélanger, G., 2007, ApJ, $662 \mathrm{~L}, 15$

Falanga, M., Melia, F., Prescher, M., Bélanger, G., \& Goldwurm, A. 2008, ApJ, 679L, 93

Falcke, H., Melia, F., \& Agol, E. 2000, ApJ, 528L, 13

Fukumura, K., Kazanas, D., \& Stephenson, G. 2009, ApJ, 695, 1199

Genzel, R., Schödel, R., Ott, T., et al. 2003, Nature, 425, 934

Ghez, A., Morris, M., Becklin, E. E., Tanner, A., \& Kremenek, T. 2000, Nature, 407, 349

Ghez, A. M., Salim, S., Hornstein, S. D., et al. 2005a, ApJ, 620, 744

Ghez, A. M., Hornstein, S. D., Lu, J. R., et al. 2005b, ApJ, 635, 1087

Gierliński, M., Middleton, M., Ward, M., \& Done, C. 2008, Nature, 455, 369 
Gillessen, S., Perrin, G., Brandner, W, et al. 2006, JPhCS, 54, 411 Gillessen, S., Eisenhauer, F., Trippe, S., et al. 2009, ApJ, 692, 1075 Goggin, L. M. 2008, PhD Thesis, California Institute of Technology Hamaus, N., Paumard, T., Müller, T., et al. 2009, ApJ, 692, 902

Hawley, J. F., \& Balbus, S. A. 1991, ApJ, 376, 223

Hirose, S., Krolik, J. H., De Villiers, J.-P., \& Hawley, J. F., 2004, ApJ, 606, 1083 Hollywood, J. M., Melia, F., Close, L. M., McCarthy, D. W. Jr., \& Dekeyser, T. A., 1995, ApJ, 448L, 21

Hornstein, S. D., Matthews, K., Ghez, A. M., et al. 2007, ApJ, 667, 900

Karas, V., \& Bao, G. 1992, A\&A, 257, 531

Karas, V., Vokrouhlicky, D., \& Polnarev, A. G. 1992, MNRAS, 259, 569

Karas V., Dovčiak M., Eckart A., \& Meyer L. 2007, in Proceedings of the Workshop on the Black Holes and Neutron Stars, ed. S. Hledík, Z. Stuchlík (Opava: Silesian University), 99 [arXiv: 0709. 3836]

Krolik, J. H., \& Hawley, J. F. 2002, ApJ, 573, 754

Kostić, U., Čadež, A., Calvani, M., \& Gomboc, A. 2009, A\&A, 496, 307

Liu, S., \& Melia, F. 2002, ApJ, 566L, 77

Liu, S., Melia, F., \& Petrosian, V. 2006, ApJ, 636, 798

Lyubarskii, Y. E. 1997, MNRAS, 292, 679

Markoff, S., Falcke, H., Yuan, F., \& Biermann, P. L. 2001, A\&A, 379, L13

Marrone, D. P., Baganoff, F. K., Morris, M., et al. 2008, ApJ, 682, 373

Mashoon, B. 1973, Phys. Rev. D 7, 2807

Melia, F. 2007, The Galactic Supermassive Black Hole (Princeton University Press)

Melia, F., Bromley, B. C., Liu, S., \& Walker, C. K. 2001, ApJ, 554, 37

Meyer, L., Eckart, A., Schödel, R., et al. 2006a, A\&A, 460, 15

Meyer, L., Schödel, R., Eckart, A., et al. 2006b, A\&A, 458, L25

Meyer, L., Schödel, R., Eckart, A., et al. 2007, A\&A, 473, 707

Mužić, K., Eckart, A., Schödel, R., Meyer, L., \& Zensus, A. 2007, A\&A, 469, 993

Nayakshin, S., Cuadra, J., \& Sunyaev, R. 2004, A\&A, 413, 173
Nishiyama, S., Tamura M., \& Hatano, H. 2009, ApJL, 702, L56

Nowak, M. A., \& Lehr, D. E. 1998, npad.conf, 233

Papa, M. A., Schutz, B. F., \& Sintes, A. M. 2000, Proceedings of the conference,

Gravitational waves: a challenge to theoretical astrophysics, ICTP Lecture Notes Series

Paumard, T., Eisenhauer, F., Genzel, R., et al. 2006, via conf., 257

Pecháček, T., Karas, V., \& Czerny, B. 2008, A\&A, 487, 815

Porquet, D., Predehl, P., Aschenbach, et al. 2003, A\&A, 407, L17

Porquet, D., Grosso, N., Predehl, P., et al. 2009, A\&A, 488, 49

Press, W. H., \& Rybicki, G. B. 1989, ApJ, 338, 277

Reid, M. J., Broderick, A. E., Loeb, A., Honma, M., \& Brunthaler, A. 2008, ApJ, 682, 1041

Schnittman, J. D., Krolik, J. H., \& Hawley, J. F., 2006, ApJ, 651, 1031

Schödel, R., Ott, T., Genzel, R., et al. 2002, Nature, 419, 694

Schödel, R., Eckart, A., Alexander, T., et al. 2007, A\&A, 469, 125

Schulz, M., \& Mudelsee, M. 2002, Computers \& Geosciences 28, 3

Tagger, M., \& Melia, F. 2006, ApJ, 636L, 33

Timmer, J., \& König, M. 1995, A\&A, 300, 707

Thorne, K. S. 1994, Black holes and time warps : Einstein's outrageous legacy (Norton \& Company)

Vaughan, S., Edelson, R., Warwick, R. S., \& Uttley, P. 2003, MNRAS, 345, 1271

Yuan, F., Markoff, S., \& Falcke, H. 2002, A\&A, 383, 854

Yuan, F., Quataert, E., \& Narayan, R. 2004, ApJ, 606, 894

Yusef-Zadeh, F., Bushouse, H., Dowell, C. D., et al. 2006a, ApJ, 644, 198

Yusef-Zadeh, F., Roberts, D., Wardle, M., Heinke, C. O., \& Bower, G. C. 2006b, ApJ, 650, 189

Yusef-Zadeh, F., Wardle, M., Cotton, W. D., Heinke, C. O., \& Roberts, D. A. 2007, ApJ, 668, 47

Yusef-Zadeh, F., Wardle, M., Heinke, C., et al. 2008, ApJ, 682, 361

Zamaninasab, M., Eckart, A., Kunneriath, D., et al. 2008a, MmSAI 79, 1054

Zamaninasab, M., Eckart, A., Meyer, L., et al. 2008b, JPhCS 131a 